\title{
Some Interior Estimates for Semidiscrete Galerkin Approximations for Parabolic Equations
}

\author{
By Vidar Thomée
}

Abstract. Consider a solution $u$ of the parabolic equation

$$
u_{t}+A u=f \quad \text { in } \quad \Omega \times[0, T]
$$

where $A$ is a second order elliptic differential operator. Let $\left\{S_{h} ; h\right.$ small $\}$ denote a family of finite element subspaces of $H^{1}(\Omega)$ which permits approximation of a smooth function to order $O\left(h^{r}\right)$. Let $\Omega_{0} \subset \Omega$ and assume that $u_{h}:[0, T] \rightarrow S_{h}$ is an approximate solution which satisfies the semidiscrete interior equation

$$
\left(u_{h, t}, x\right)+A\left(u_{h}, x\right)=(f, x) \quad \forall x \in s_{h}^{0}\left(\Omega_{0}\right)=\left\{x \in S_{h}, \operatorname{supp} x \subset \Omega_{0}\right\},
$$

where $A(\cdot, \cdot)$ denotes the bilinear form on $H^{1}(\Omega)$ associated with $A$. It is shown that if the finite element spaces are based on uniform partitions in a specific sense in $\Omega_{0}$, then difference quotients of $u_{h}$ may be used to approximate derivatives of $u$ in the interior of $\Omega_{0}$ to order $O\left(h^{r}\right)$ provided certain weak global error estimates for $u_{h}-u$ to this order are available. This generalizes results proved for elliptic problems by Nitsche and Schatz [9] and Bramble, Nitsche and Schatz [1].

1. Introduction. Let $A$ be a second order elliptic differential operator in a smooth domain $\Omega \subset R^{N}$ and let $u$ be a solution of

$$
A u=f \quad \text { in } \Omega,
$$

with some boundary condition on $\partial \Omega$. Let $\left\{S_{h} ; h\right.$ small $\}$ denote a family of finite element subspaces of $H^{1}(\Omega)$ which permits approximation of a smooth function to order $O\left(h^{r}\right)$ in $L_{2}$ or $L_{\infty}$, say. Let $\Omega_{0} \subset \Omega$ and assume that $u_{h} \in S_{h}$ is an approximate solution of (1.1) which satisfies the interior equation

$$
A\left(u_{h}, \chi\right)=(f, \chi) \quad \forall \chi \in S_{h}^{0}\left(\Omega_{0}\right)=\left\{\chi \in S_{h} ; \operatorname{supp} \chi \subset \Omega_{0}\right\},
$$

where $A(\cdot, \cdot)$ denotes the bilinear form on $H^{1}(\Omega)$ associated with the operator $A$. It was then proved in Nitsche and Schatz [9], Bramble, Nitsche and Schatz [1] that if the finite element spaces are based on uniform partitions in a specific sense in the interior domain $\Omega_{0}$, then difference quotients of $u$ may be used to approximate derivatives of $u$ in the interior of $\Omega_{0}$ to order $O\left(h^{r}\right)$. More precisely, it was shown in [1] that if $Q_{h}$ is a finite difference operator approximating $D^{\alpha}(|\alpha|=m)$ with accuracy $r$, if $\Omega_{2} \subset \subset \Omega_{1} \subset \Omega_{0}$ and if $u$ is sufficiently smooth, then for each $p$,

Received November 14, 1977.

AMS (MOS) subject classifications (1970). Primary 65N15, 65N30. 


$$
\begin{gathered}
\left|Q_{h} u_{h}-D^{\alpha} u\right|_{\Omega_{2}} \leqslant C h^{r}\|u\|_{m+r+N_{0}, \Omega_{1}}+C\left\|u_{n}-u\right\|_{-p, \Omega_{1}}, \\
N_{0}=[N / 2]+1 .
\end{gathered}
$$

Combined with known global error estimates for $u_{h}$ this yields uniform interior estimates for approximations to arbitrary derivatives. Here and below we denote for $\Omega_{1}$ $\subset \Omega$,

$$
|\cdot|_{m, \Omega_{1}}=\|\cdot\|_{W_{\infty}^{m}\left(\Omega_{1}\right)} \text { and }\|\cdot\|_{m, \Omega_{1}}=\|\cdot\|_{H^{m}\left(\Omega_{1}\right)},
$$

with $m$ and $\Omega_{1}$ suppressed when $m=0$ or $\Omega_{1}=\Omega$, respectively, and where for $m>$ 0 with $(\cdot, \cdot)$ the inner product in $L_{2}(\Omega)$,

$$
\|u\|_{H^{-m}\left(\Omega_{1}\right)}=\sup _{\varphi \in C_{0}^{\infty}\left(\Omega_{1}\right)} \frac{(u, \varphi)}{\|\varphi\|_{H^{m}\left(\Omega_{1}\right)}} .
$$

The purpose of the present paper is to derive similar results for the solution $u$ of a parabolic equation $\left(u_{t}=\partial u / \partial t\right)$

$$
u_{t}+A u=f \quad \text { in } \Omega \times[0, T],
$$

and an approximate solution $u_{h}:[0, T] \rightarrow S_{h}$ satisfying the semidiscrete interior equation

$$
\left(u_{h, t}, \chi\right)+A\left(u_{h}, \chi\right)=(f, \chi) \quad \forall \chi \in S_{h}^{0}\left(\Omega_{0}\right) .
$$

These results are stated and proved in Section 4. Our first main result (Theorem 4.1 below) shows that similarly to (1.2) above, if the initial-values satisfy, with $A_{0}(\cdot, \cdot)$ some elliptic bilinear form on $H^{1}(\Omega)$,

$$
A_{0}\left(u_{h}(0)-u(0), \chi\right)=0 \quad \forall \chi \in S_{h}^{0}\left(\Omega_{0}\right),
$$

then for $t \in[0, T]$, and with $e_{h}=u_{h}-u$,

$$
\left|Q_{h} u_{h}(t)-D^{\alpha} u(t)\right|_{\Omega_{2}} \leqslant C\left\{h^{r} B(u)+R\left(e_{h}\right)\right\},
$$

where $B(u)$ depends on a number of derivatives of $u$ in $\Omega_{1} \times[0, t]$ and $R\left(e_{h}\right)$ consists of weak norms on this set of the error; with $p, q$ arbitrary numbers,

$$
R\left(e_{h}\right)=\left\|e_{h}(0)\right\|_{-p, \Omega_{1}}+\left(\int_{0}^{t}\left(\left\|e_{h}\right\|^{2}+h^{q}\left\|e_{h, t}\right\|^{2}\right) d \tau\right)^{1 / 2} .
$$

In our second main result (Theorem 4.2) we show that if we are content with an estimate for time bounded away from zero, then an error estimate analogous to (1.4) holds, with $B(u)$ and $R\left(e_{h}\right)$ now only depending on $u$ and $e_{h}$ over a short interval preceding $t$ and without any restriction on the initial-values. The first of these two results may be thought of as interior in space only whereas the second is then interior with respect to both space and time. In the same way as for the elliptic problem quoted above, these estimates may be combined with known estimates for $e_{h}$ to yield spatially interior uniform $O\left(h^{r}\right)$ bounds for $Q_{h} u_{h}-D^{\alpha} u$. Several such error estimates are available in the literature, cf. e.g. [3], [5], [6], [7], [10], [11], and further 
estimates, specially tailored to the present situation, are derived in Section 5 below.

An estimate for $Q_{h} u_{h}-D^{\alpha} u$ of the type just described was obtained in Bramble, Schatz, Thomée and Wahlbin [2] as a part of a rather general theory treating the homogeneous case $(f=0)$ of the equation (1.3) under Dirichlet boundary conditions, with $A$ selfadjoint, nonnegative and time-independent.

This particular estimate was derived using the corresponding known estimates in the elliptic case quoted above, by considering $u_{h}$ as an approximate solution of a nonhomogeneous elliptic problem with $u_{t}$ as a right-hand side, first trading difference quotients in space for time derivatives and then applying global error estimates for time derivatives. These global estimates were obtained by a method using spectral representations and allowing for considerable generality in treating the approximation of the boundary conditions. We shall review the framework of these estimates in Section 5 below. The constant in the resulting $O\left(h^{r}\right)$ estimate for $Q_{h} u_{h}-D^{\alpha} u$ here behaves like a negative power of $t$ and the estimate, therefore, is not valid uniformly for small $t$. Also, since it uses global error estimates in space for the time derivatives, restrictive regularity assumptions have to be imposed on the solution which are global in space. The technique of estimating $Q_{h} u_{h}-D^{\alpha} u$ in terms of time derivatives of $e_{h}$ generalizes to the nonhomogeneous equation as stated in Theorem 4.3.

Besides being valid for the nonhomogeneous equation the error estimates of the present paper differ from the corresponding ones of [2] in that they require severe regularity assumptions only in a neighborhood of the domain over which the error is sought, and one of the estimates is uniform down to $t=0$. They will be derived by the energy method, using the parabolic character directly rather than considering $u_{t}$ as an inhomogeneity in an elliptic equation. This method of proof will allow us to treat without complications operators which are nonselfadjoint and which depend on time. The major step is to derive a spatially interior estimate for the semidiscrete parabolic equation corresponding to the following estimate for the continuous problem, namely

$$
\|u(t)\|_{m, \Omega_{2}} \leqslant C\left\{\|u(0)\|_{m, \Omega_{1}}+\left(\int_{0}^{t}\left(\|f\|_{m-1, \Omega_{1}}^{2}+\|u\|_{\Omega_{1}}^{2}\right) d \tau\right)^{1 / 2}\right\} .
$$

This result will be shown in Section 3 below. One of the tools in deriving it is a super-approximation result for the approximating spaces which is based on the following super-approximation property assumed in [1] and [9]: for $\omega \in C_{0}^{\infty}\left(\Omega_{1}\right)$,

$$
\inf _{\chi \in S_{h}^{0}\left(\Omega_{1}\right)}\|\omega U-\chi\|_{1, \Omega_{1}} \leqslant C h\|U\|_{1, \Omega_{1}} \quad \forall U \in S_{h} .
$$

The result we shall need (Lemma 2.4 below) is essentially that if $\widetilde{P}: H^{1}\left(\Omega_{1}\right) \rightarrow S_{h}$ denotes the elliptic projection over $\Omega_{1}$ with respect to $A(\cdot, \cdot)$, without boundary conditions, then

$$
\inf _{x \in S_{h}^{0}\left(\Omega_{1}\right)}\|\tilde{P}(\omega U)-\chi\|_{1, \Omega_{1}} \leqslant C h\|U\|_{\Omega_{1}} .
$$

Notice that the norm on the right in (1.6) is weaker than that in (1.5). 
As indicated above, Section 5 is devoted to deriving, within the framework of the nonhomogeneous analogue of the theory developed in [2] the global $O\left(h^{r}\right)$ estimates needed for appraising to this order the terms in $e_{h}$ in the results of Section 4 . The first such result, Theorem 5.1, corresponding to the situation of Theorem 4.1, estimates the term $R\left(e_{h}\right)$ in (1.4) with $u_{h}(0)$ chosen as the elliptic projection of $u(0)$ and under certain regularity assumptions on $u$ in $\Omega_{1} \times[0, t]$. In Theorem 5.2 we then show, using the results of [2] that if, as in Theorem 4.2, we are content with error estimates for $t$ bounded away from zero, then those regularity assumptions only have to be made for the time immediately preceding $t$. We shall also see that in this case we have considerable freedom in the choice of the discrete initial-values. For completeness we finally deduce in Theorem 5.3 the global estimates for the time derivatives of the error needed to generalize the argument of [2] to the nonhomogeneous equation for time bounded away from zero. Again, this method uses more than necessary regularity, globally in space.

Throughout this paper $C$ will denote different positive constants, independent of $h, t$ and the functions involved.

2. Interior Approximation Properties and Elliptic Estimates. In this section we shall first briefly recall the local regularity assumptions on the finite element spaces $S_{h}$ employed in Nitsche and Schatz [9] and Bramble, Nitsche and Schatz [1], and review some of the interior estimates of these papers which will be needed below. We shall then introduce a local elliptic projection which when applied to the exact solution of the parabolic problem will be shown in Section 4, as a major step towards our final results, to yield a function in $S_{h}$ close to the semidiscrete solution. We shall finally show a super-approximation result for the local elliptic projection of a localization of a function in $S_{h}$.

We begin with the assumptions on $\left\{S_{h}\right\}$ and postulate first that for some interior subdomain $\Omega_{0}$ of $\Omega$, the functions in $S_{h}$ are piecewise polynomials on a uniform partition. In order to make this more precise, let $Q_{1}, \ldots, Q_{l}$ be disjoint bounded domains in $R^{N}$ such that their translates $Q_{j}^{\nu}=Q_{j}+\nu, j=1, \ldots, l, \nu \in Z^{N}$, are disjoint and their closures cover $R^{N}$. Let $r$ be an integer $\geqslant 2$ and let $\psi_{1}, \ldots, \psi_{k}$ be continuous functions with compact supports which reduce to polynomials on the sets $Q_{j}^{\nu}$ and which are such that for each $Q_{j}^{\nu}$ the set of restrictions to $Q_{j}^{\nu}$ of $\left\{\psi_{s}(\cdot-\alpha)\right.$; $\left.s=1, \ldots, k, \alpha \in Z^{N}\right\}$ contains all polynomials of degree less than $r$. We shall assume then that $S_{h}\left(\Omega_{0}\right)$, the set of restrictions to $\Omega_{0}$ of the functions in $S_{h}$, is spanned by the translates of the $\psi_{j}$, scaled to mesh-size $h$, or $\chi \in S_{h}\left(\Omega_{0}\right)$ if and only if it can be written in the form

$$
\chi(x)=\sum_{j, \alpha} a_{j \alpha} \psi_{j}\left(h^{-1} x-\alpha\right) \text { for } x \in \Omega_{0} .
$$

We note that as a consequence of this assumption, if $\Omega_{1} \subset \subset \Omega_{0}$, then for small $h$ any finite difference quotient with step-size $h$ of a function in $S_{h}\left(\Omega_{0}\right)$ is in $S_{h}\left(\Omega_{1}\right)$. Further, for any $\Omega_{h} \subset \Omega_{0}$ which is a mesh-domain (the interior of the closure of a union of some sets $h Q_{j}^{\nu}$ ) we have the inverse estimate (clearly $S_{h}\left(\Omega_{0}\right) \subset H^{1}\left(\Omega_{0}\right)$ ) 
(a)

$$
\|\chi\|_{1, \Omega_{h}} \leqslant C h^{-1}\|\chi\|_{\Omega_{h}} \quad \forall \chi \in S_{h}\left(\Omega_{0}\right) .
$$

We shall make the following two local approximability assumptions, where for $\Omega_{1} \subset \Omega_{0}$,

$$
S_{h}^{0}\left(\Omega_{1}\right)=\left\{\chi \in S_{h} ; \operatorname{supp} \chi \subset \Omega_{1}\right\},
$$

and where $\Omega_{2} \subset \subset \Omega_{1} \subset \Omega_{0}$. The first assumption concerns the possibility of approximating functions with compact support in $\Omega_{1}$ by functions in $S_{h}^{0}\left(\Omega_{1}\right)$ :

(b) If $1 \leqslant l \leqslant r$ and if $w \in H^{l}\left(\Omega_{1}\right)$ and vanishes outside $\Omega_{2}$, then

$$
\inf _{\chi \in S_{h}^{0}\left(\Omega_{1}\right)}\|w-\chi\|_{1, \Omega_{1}} \leqslant C h^{l-1}\|w\|_{l, \Omega_{1}} .
$$

The second assumption is essential in order to be able to localize functions in $S_{h}$ :

(c) If $\omega \in C^{\infty}\left(\bar{\Omega}_{1}\right)$ with supp $\nabla \omega \subset \Omega_{2}$, then for $U \in S_{h}\left(\Omega_{1}\right)$,

$$
\inf _{\substack{x \in S_{h}\left(\Omega_{1}\right) \\ \chi=\omega U \text { in } \Omega_{1} \backslash \Omega_{2}}}\|\omega U-\chi\|_{1, \Omega_{1}} \leqslant C\|U\|_{\Omega_{1}} .
$$

For a more detailed discussion of these properties we refer to [9] and [1]. It is in fact only the properties (a) and (c) which will be used explicitly below; the others are made in order to permit application of the elliptic interior estimates of [9] and [1]. In these papers, the latter property was stated only for $\omega \in C_{0}^{\infty}\left(\Omega_{1}\right)$ and in the form

$$
\inf _{\chi \in S_{h}^{0}\left(\Omega_{1}\right)}\|\omega U-\chi\|_{1, \Omega_{1}} \leqslant C h\|U\|_{1, \Omega_{1}}
$$

which then implies the estimate in (c) in view of (a). In the examples discussed in [9] and [1] one finds easily that (2.1) is valid for the more general $\omega$ used in (c). In many cases, an interpolant of $\omega U$ may be used to show (c).

Let now $A$ be the elliptic operator

$$
A u=-\sum_{j, k=1}^{N} \frac{\partial}{\partial x_{j}}\left(a_{j k} \frac{\partial u}{\partial x_{k}}\right)+\sum_{j=1}^{N} a \frac{\partial u}{j \partial x_{j}}+a_{0} u
$$

where we assume that the coefficients are in $C^{\infty}(\bar{\Omega} \times[0, T])$ and $\left(a_{j k}\right)$ is uniformly positive definite in $\bar{\Omega} \times[0, T]$. We introduce the corresponding bilinear form

$$
A(v, w)=A(t ; v, w)=\int_{\Omega}\left(\sum_{j, k=1}^{N} a_{j k} \frac{\partial v}{\partial x_{k}} \frac{\partial w}{\partial x_{j}}+\sum_{j=1}^{N} a_{j} \frac{\partial v}{\partial x_{j}} w+a_{0} v w\right) d x
$$

We shall quote some interior estimates for discrete elliptic equations which we shall need. We shall always assume that our above assumptions on $S_{h}$ hold in $\Omega_{0} \subset \subset$ $\Omega$, although the full force of these are not required in each instance. Notice in particular that the constants below are independent of $t$.

Lемма 2.1. Let $\Omega_{1} \subset \Omega_{0}$ and assume that $w_{h} \in S_{h}$ satisfies

$$
A\left(t ; w_{h}, \chi\right)=0 \quad \forall \chi \in S_{h}^{0}\left(\Omega_{1}\right) .
$$


Then for any $\Omega_{2} \subset \subset \Omega_{1}$,

$$
\left\|w_{h}\right\|_{1, \Omega_{2}} \leqslant C\left\|w_{h}\right\|_{\Omega_{1}} .
$$

Proof. See [9, Lemma 5.2].

In the next lemma we denote by $\partial_{h}^{\alpha}$ the forward difference quotient

$$
\partial_{h}^{\alpha}=\partial_{h, 1}^{\alpha_{1}} \cdots \partial_{h, N}^{\alpha, N} \quad \text { with } \quad \partial_{h, j} w(x)=h^{-1}\left(w\left(x+h e_{j}\right)-w(x)\right),
$$

where $e_{j}$ is the $j$ th unit vector in $R^{N}$.

LEMMA 2.2. Let $\Omega_{1} \subset \Omega_{0}$ and assume that $w_{h} \in S_{h}$ satisfies

$$
A\left(t ; w_{h}-w, \chi\right)=0 \quad \forall \chi \in S_{h}^{0}\left(\Omega_{1}\right) .
$$

Then for any $\Omega_{2} \subset \subset \Omega_{1}$ and $p \geqslant 0,|\alpha|=m$,

$$
\left\|\partial_{h}^{\alpha}\left(w_{h}-w\right)\right\|_{\Omega_{2}} \leqslant C\left\{h^{r}\|w\|_{r+m, \Omega_{1}}+\left\|w_{h}-w\right\|_{-p, \Omega_{1}}\right\}
$$

and with $N_{0}=[N / 2]+1$,

$$
\left|\partial_{h}^{\alpha}\left(w_{h}-w\right)\right|_{\Omega_{2}} \leqslant C\left\{h^{r}\|w\|_{r+m+N_{0}, \Omega_{1}}+\left\|w_{h}-w\right\|_{-p, \Omega_{1}}\right\} .
$$

Further, for the time derivative of the error,

$$
\begin{aligned}
\left\|\partial_{h}^{\alpha}\left(w_{h}-w\right)_{t}\right\|_{\Omega_{2}} \leqslant C\{ & h^{r}\left(\|w\|_{r+m, \Omega_{1}}+\left\|w_{t}\right\|_{r+m, \Omega_{1}}\right) \\
& \left.+\left\|w_{h}-w\right\|_{-p, \Omega_{1}}+\left\|\left(w_{h}-w\right)_{t}\right\|_{-p, \Omega_{1}}\right\} .
\end{aligned}
$$

Proof. The estimates (2.2) and (2.3) are contained in [9, Theorem 6.1] and [1, Theorem 1], respectively, and (2.4) follows in the same way as (2.2) using [9, Theorem 5.2] and the fact that

$$
A\left(t ;\left(w_{h}-w\right)_{t}, \chi\right)=-A^{\prime}\left(t ; w_{h}-w, \chi\right) \quad \forall \chi \in S_{h}^{0}\left(\Omega_{1}\right),
$$

with $A^{\prime}$ the bilinear form obtained from $A$ by time-differentiation of the coefficients. When $A$ is independent of $t,(2.4)$ is of course an immediate consequence of (2.2) and the first and third terms on the right may then be omitted.

We shall have reason to work below with a local elliptic projection corresponding to a Neumann problem on a subdomain $\Omega_{1}$ of $\Omega_{0}$ which we may assume to be smooth. For this purpose we denote

$$
\widetilde{A}_{\Omega_{1}}(t ; v, w)=\int_{\Omega_{1}}\left(\sum_{j, k=1}^{N} a_{j k} \frac{\partial v}{\partial x_{k}} \frac{\partial w}{\partial x_{j}}+v w\right) d x .
$$

Note that this modified symmetric bilinear form is $H^{1}\left(\Omega_{1}\right)$-elliptic, uniformly in $t$, so that

$$
\|v\|_{1, \Omega_{1}}^{2} \leqslant C \tilde{A}_{\Omega_{1}}(t ; v, v) \quad \forall v \in H^{1}\left(\Omega_{1}\right), 0 \leqslant t \leqslant T .
$$

We then define $\widetilde{P}_{\Omega_{1}}(t): H^{1}\left(\Omega_{1}\right) \rightarrow S_{h}\left(\Omega_{1}\right)$ by

$$
\widetilde{A}_{\Omega_{1}}\left(t ; w-\widetilde{P}_{\Omega_{1}}(t) w, \chi\right)=0 \quad \forall \chi \in S_{h}\left(\Omega_{1}\right) .
$$


It is well known that

$$
\left\|\left(I-\widetilde{P}_{\Omega_{1}}(t)\right) w\right\|_{j, \Omega_{1}} \leqslant C h^{l-j}\|w\|_{l, \Omega_{1}}, \quad-(r-2) \leqslant j \leqslant 1 \leqslant l \leqslant r
$$

and similarly for the time derivative, in particular (cf. e.g. Douglas and Dupont [4]),

$$
\left\|\left(\left(I-\widetilde{P}_{\Omega_{1}}(t)\right) w\right)_{t}\right\|_{\Omega_{1}} \leqslant C h^{l}\left(\|w\|_{l, \Omega_{1}}+\left\|w_{t}\right\|_{l, \Omega_{1}}\right), \quad 1 \leqslant l \leqslant r .
$$

Combining these estimates with Lemma 2.2, we have

Lemma 2.3. Let $\Omega_{1} \subset \Omega_{0}$ and let $\widetilde{P}=\widetilde{P}_{\Omega_{1}}(t)$ be defined by (2.5). Then for $\Omega_{2} \subset \subset \Omega_{1}$ and $|\alpha|=m$,

$$
\begin{aligned}
& \left\|\partial_{h}^{\alpha}(I-\widetilde{P}) w\right\|_{\Omega_{2}} \leqslant C h^{r}\|w\|_{r+m, \Omega_{1}}, \\
& \left|\partial_{h}^{\alpha}(I-\widetilde{P}) w\right|_{\Omega_{2}} \leqslant C h^{r}\|w\|_{r+m+N_{0}, \Omega_{1}},
\end{aligned}
$$

and

$$
\left\|\partial_{h}^{\alpha}((I-\widetilde{P}) w)_{t}\right\|_{\Omega_{2}} \leqslant C h^{r}\left(\|w\|_{r+m, \Omega_{1}}+\left\|w_{t}\right\|_{r+m, \Omega_{1}}\right) .
$$

We conclude this section by establishing our super-approximation estimate for the elliptic projection defined above of a localization of a function in the subspace. We emphasize that this estimate shows one degree better approximation than the corresponding estimates (c) and (2.1).

LEMmA 2.4. Let $\Omega_{1} \subset \Omega_{0}$ and $\omega \in C_{0}^{\infty}\left(\Omega_{1}\right)$. Then for $U \in S_{h}\left(\Omega_{1}\right)$,

$$
\inf _{\chi \in S_{h}^{0}\left(\Omega_{1}\right)}\left\{\left\|\widetilde{P}_{\Omega_{1}}(t)(\omega U)-\chi\right\|_{1, \Omega_{1}}+\|\omega U-\chi\|_{\Omega_{1}}\right\} \leqslant C h\|U\|_{\Omega_{1}} .
$$

Proof. Set for brevity $\widetilde{U}=\widetilde{P}_{\Omega_{1}}(t)(\omega U)$ and $\widetilde{A}(\cdot, \cdot)=\widetilde{A}_{\Omega_{1}}(t ; \cdot, \cdot)$. We shall prove below that

$$
\inf _{\chi \in S_{h}^{0}\left(\Omega_{1}\right)}\|\widetilde{U}-\chi\|_{1, \Omega_{1}} \leqslant C h\|U\|_{\Omega_{1}} .
$$

Let us first show that this implies (2.8). In fact,

$$
\begin{gathered}
\inf _{\chi \in S_{h}^{0}\left(\Omega_{1}\right)}\left(\|\widetilde{U}-\chi\|_{1, \Omega_{1}}+\|\omega U-\chi\|_{\Omega_{1}}\right) \\
\leqslant\|\omega U-\widetilde{U}\|_{\Omega_{1}}+C \inf _{\chi \in S_{h}^{0}\left(\Omega_{1}\right)}\|\widetilde{U}-\chi\|_{1, \Omega_{1}},
\end{gathered}
$$

so that with (2.9) proven it suffices to show

$$
\|\omega U-\widetilde{U}\|_{\Omega_{1}} \leqslant C h\|U\|_{\Omega_{1}}
$$

In order to prove $(2.10)$ we note that duality yields

$$
\|\omega U-\widetilde{U}\|_{\Omega_{1}} \leqslant C h\|\omega U-\widetilde{U}\|_{1, \Omega_{1}} \leqslant C h \widetilde{A}(\omega U-\widetilde{U}, \omega U-\widetilde{U})^{1 / 2},
$$


and hence by the definition of $\widetilde{U}$ as the best approximation of $\omega U$ with respect to $\widetilde{A}(\cdot, \cdot)^{1 / 2}$, and by $(\mathrm{c})$,

$$
\|\omega U-\tilde{U}\|_{\Omega_{1}} \leqslant C h \inf _{\chi \in S_{h}^{0}\left(\Omega_{1}\right)}\|\omega U-\chi\|_{1, \Omega_{1}} \leqslant C h\|U\|_{\Omega_{1}} .
$$

In order to show (2.9) we shall only need to show that

$$
\inf _{x \in S_{h}^{0}\left(\Omega_{1}\right)}\|\tilde{U}-\chi\|_{1, \Omega_{1}} \leqslant C\|\tilde{U}\|_{\Omega_{1} \backslash \operatorname{supp} \omega} .
$$

For by (2.10) we have

$$
\|\tilde{U}\|_{\Omega_{1} \backslash \operatorname{supp} \omega} \leqslant\|\widetilde{U}-\omega U\|_{\Omega_{1}} \leqslant C h\|U\|_{\Omega_{1}}
$$

and together (2.11) and (2.12) prove (2.9).

For the purpose of showing (2.11), let $\Omega_{2}$ and $\Omega_{3}$ be such that supp $\omega \subset \Omega_{3}$ $\subset \subset \Omega_{2} \subset \subset \Omega_{1}$. We shall then first prove that

$$
\inf _{\chi \in S_{h}^{0}\left(\Omega_{1}\right)}\|\widetilde{U}-\chi\|_{1, \Omega_{1}} \leqslant C\|\widetilde{U}\|_{1, \Omega_{1} \backslash \bar{\Omega}_{2}}
$$

Since $\widetilde{U}$ is the elliptic projection of a function vanishing on $\Omega_{1} \mid \bar{\Omega}_{2}$, we shall be able to estimate its norm in $H^{1}\left(\Omega_{1} \mid \bar{\Omega}_{2}\right)$ by a norm in $L_{2}$. In order, however, to be able to apply the interior estimate of Lemma 2.1 we shall first shift the problem into the interior of $\Omega_{1}$ by showing below that

$$
\|\widetilde{U}\|_{1, \Omega_{1} \backslash \bar{\Omega}_{2}} \leqslant C\|\widetilde{U}\|_{1, \Omega_{2} \backslash \bar{\Omega}_{3}}
$$

We now note that by the definition of $\widetilde{U}$ we have

$$
\widetilde{A}(\widetilde{U}, \chi)=\widetilde{A}(\omega U, \chi)=0 \quad \forall \chi \in S_{h}^{0}\left(\Omega_{1} \backslash \operatorname{supp} \omega\right),
$$

so that Lemma 2.1 yields

$$
\|\widetilde{U}\|_{1, \Omega_{2} \backslash \bar{\Omega}_{3}} \leqslant C\|\widetilde{U}\|_{\Omega_{1} \backslash \text { supp } \omega} .
$$

Together, (2.13), (2.14) and (2.15) imply (2.11) and thus complete the proof of (2.9) and hence of the lemma.

It remains to show (2.13) and (2.14). In order to show (2.13), let $\omega_{1} \in C_{0}^{\infty}\left(\Omega_{1}\right)$ with $\omega_{1}=1$ in a neighborhood of $\Omega_{2}$. Then

$$
\inf _{\chi \in S_{h}^{0}\left(\Omega_{1}\right)}\|\widetilde{U}-\chi\|_{1, \Omega_{1}} \leqslant\left\|\left(1-\omega_{1}\right) \widetilde{U}\right\|_{1, \Omega_{1}}+\inf _{\chi \in S_{h}^{0}\left(\Omega_{1}\right)}\left\|\omega_{1} \widetilde{U}-\chi\right\|_{1, \Omega_{1}} .
$$

Since $\operatorname{supp}\left(1-\omega_{1}\right) \subset \Omega_{1} \backslash \bar{\Omega}_{2}$ we have for the first term,

$$
\left\|\left(1-\omega_{1}\right) \widetilde{U}\right\|_{1, \Omega_{1}} \leqslant C\|\widetilde{U}\|_{1, \Omega_{1} \backslash \bar{\Omega}_{2}} .
$$

For the second term, letting $\Omega_{4}$ be such that supp $\nabla \omega_{1} \subset \Omega_{4} \subset \subset \Omega_{1} \backslash \bar{\Omega}_{2}$ : we obtain, using in the last step (c) applied to $\Omega_{1} \backslash \bar{\Omega}_{2}$, 


$$
\begin{gathered}
\inf _{x \in S_{h}^{0}\left(\Omega_{1}\right)}\left\|\omega_{1} \widetilde{U}-\chi\right\|_{1, \Omega_{1}} \leqslant \inf _{\substack{x \in S_{h}\left(\Omega_{1}\right) \\
\chi=\omega_{1} \widetilde{U} \text { in } \Omega_{1} \backslash \Omega_{4}}}\left\|\omega_{1} \widetilde{U}-\chi\right\|_{1, \Omega_{1}} \\
=\inf _{\substack{x \in S_{h}\left(\Omega_{1} \backslash \bar{\Omega}_{2}\right) \\
x=\omega_{1} \widetilde{U} \text { in }\left(\Omega_{1} \backslash \bar{\Omega}_{2}\right) \backslash \bar{\Omega}_{4}}}\left\|\omega_{1} \widetilde{U}-\chi\right\|_{1, \Omega_{1} \backslash \bar{\Omega}_{2}} \leqslant C\|\widetilde{U}\|_{\Omega_{1} \backslash \bar{\Omega}_{2}} .
\end{gathered}
$$

Together these estimates show (2.13).

We finally turn to (2.14). Let $\varphi \in C^{\infty}\left(\Omega_{1}\right)$ with $\varphi=1$ in a neighborhood of $\Omega_{1} \backslash \Omega_{2}$ in $\Omega_{1}$ and $\varphi=0$ in a neighborhood of $\bar{\Omega}_{3}$. Let also $\Omega_{5}$ be such that supp $\nabla \varphi \subset \Omega_{5} \subset \subset \Omega_{2} \backslash \bar{\Omega}_{3}$. We have

$$
\begin{aligned}
\|\widetilde{U}\|_{1, \Omega_{1} \backslash \Omega_{2}}^{2} & \leqslant\|\varphi \widetilde{U}\|_{1, \Omega_{1}}^{2} \leqslant C \widetilde{A}(\varphi \widetilde{U}, \varphi \widetilde{U}) \\
& =C\left\{\widetilde{A}\left(\widetilde{U}, \varphi^{2} \widetilde{U}\right)+\left[A(\varphi \widetilde{U}, \varphi \widetilde{U})-\widetilde{A}\left(\widetilde{U}, \varphi^{2} \widetilde{U}\right)\right]\right\} .
\end{aligned}
$$

Here, for $\chi \in S_{h}\left(\Omega_{1}\right)$ with $\chi=\varphi^{2} \widetilde{U}$ outside $\Omega_{5}$ we have

$$
\widetilde{A}\left(\widetilde{U}, \varphi^{2} \widetilde{U}\right)=\widetilde{A}\left(\widetilde{U}, \varphi^{2} \widetilde{U}-\chi\right) \leqslant C\|\widetilde{U}\|_{1, \Omega_{2} \backslash \Omega_{3}}\left\|\varphi^{2} \widetilde{U}-\chi\right\|_{1, \Omega_{2} \backslash \bar{\Omega}_{3}},
$$

so that by (c),

$$
\begin{aligned}
\widetilde{A}\left(\widetilde{U}, \varphi^{2} \widetilde{U}\right) & \leqslant C\|\widetilde{U}\|_{1, \Omega_{2} \backslash \bar{\Omega}_{3}} \inf _{\substack{\chi \in S_{h}\left(\Omega_{2} \backslash \bar{\Omega}_{3}\right) \\
\chi=\varphi^{2} \widetilde{U} \text { in }\left(\Omega_{2} \backslash \bar{\Omega}_{3}\right) \backslash \Omega_{5}}}\left\|\varphi^{2} \widetilde{U}-\chi\right\|_{1, \Omega_{2} \backslash \bar{\Omega}_{3}} \\
& \leqslant C\|\widetilde{U}\|_{1, \Omega_{2} \backslash \bar{\Omega}_{3}}^{2} .
\end{aligned}
$$

Further, by an easy calculation,

$$
\begin{aligned}
\tilde{A}(\varphi \widetilde{U}, \varphi \widetilde{U})-\widetilde{A}\left(\widetilde{U}, \varphi^{2} \widetilde{U}\right) \mid & =\left|\int_{\Omega_{1}} \sum_{j, k} a_{j k} \frac{\partial \varphi}{\partial x_{j}} \frac{\partial \varphi}{\partial x_{k}} \widetilde{U}^{2} d x\right| \\
& \leqslant C\|\widetilde{U}\|_{\operatorname{sup~}(\nabla \varphi)}^{2} \leqslant C\|\widetilde{U}\|_{1, \Omega_{2} \backslash \bar{\Omega}_{3}}^{2}
\end{aligned}
$$

Together, (2.16), (2.17) and (2.18) yield (2.14).

The proof of Lemma 2.4 is now complete.

3. Interior Estimates for the Semidiscrete Problem. In this section we shall first derive, in Lemma 3.1, a discrete interior version of the energy type inequality

$$
\|u(t)\|^{2}+\int_{0}^{t}\|u\|_{1}^{2} d \tau \leqslant C\left\{\|u(0)\|^{2}+\int_{0}^{t}\|f\|_{-1}^{2} d \tau\right\}
$$

valid for a solution $u$ of the continuous equation (1.1), vanishing on $\partial \Omega$. We shall then apply this inequality to difference quotients to obtain our basic a priori interior estimate, which is given in Lemma 3.3 below. The proof of the discrete interior counterpart of (3.1) will depend on the super-approximation property, described in Lemma 2.4 , of the projection $\widetilde{P}_{\Omega_{1}^{\prime}}(t)$ defined by (2.5), with $\Omega_{1}^{\prime}$ a subdomain of $\Omega_{0}$. 
We define for $U:[0, T] \rightarrow S_{h}\left(\Omega_{0}\right)$,

$$
\left(L_{h} U\right)(\chi)=\left(U_{t}, \chi\right)+A(t ; U, \chi) \text { for } \chi \in S_{h}\left(\Omega_{0}\right),
$$

and set for $\Omega_{1} \subset \Omega_{0}$,

$$
\left\|L_{h} U\right\|_{h,-j, \Omega_{1}}=\sup _{\chi \in S_{h}^{0}\left(\Omega_{1}\right)} \frac{\left|\left(L_{h} U\right)(\chi)\right|}{\|\chi\|_{j, \Omega_{1}}}, \quad j=0,1 .
$$

We have then

LemmA 3.1. Let $\Omega_{2} \subset \subset \Omega_{1} \subset \Omega_{0}$ and let $q$ be an arbitrary number. We then have for $U:[0, T] \rightarrow S_{h}\left(\Omega_{0}\right)$,

$$
\begin{aligned}
\|U(t)\|_{\Omega_{2}}^{2} & +\int_{0}^{t}\|U\|_{1, \Omega_{2}}^{2} d \tau \\
& \leqslant C\left\{\|U(0)\|_{\Omega_{1}}^{2}+\int_{0}^{t}\left[\|U\|_{\Omega_{1}}^{2}+h^{q}\left\|U_{t}\right\|_{\Omega_{1}}^{2}+\left\|L_{h} U\right\|_{h,-1, \Omega_{1}}^{2}\right] d \tau\right\} .
\end{aligned}
$$

Proof. We shall first show this result for $q=2$. We shall then derive in Lemma 3.2 an estimate for $U_{t}$ which will allow us to improve the result to the case of a general $q$.

Let $\Omega_{2} \subset \subset \Omega_{1}^{\prime} \subset \subset \Omega_{1}$, take $\omega \in C_{0}^{\infty}\left(\Omega_{1}^{\prime}\right)$ with $\omega \equiv 1$ on $\Omega_{2}$ and let $\widetilde{P}=$ $\widetilde{P}_{\Omega_{1}^{\prime}}(t)$ be the projection defined above with respect to $\widetilde{A}(\cdot, \cdot)=\widetilde{A}_{\Omega_{1}^{\prime}}(t ; \cdot, \cdot)$. Then using the definition of $L_{h} U$ we have for any $\chi \in S_{h}^{0}\left(\Omega_{1}^{\prime}\right)$,

$$
\begin{aligned}
\left(U_{t}, \omega^{2} U\right) & +\widetilde{A}\left(U, \omega^{2} U\right) \\
= & \left(U_{t}, \omega^{2} U-\chi\right)+\widetilde{A}\left(U, \widetilde{P}\left(\omega^{2} U\right)-\chi\right)+\left(L_{h} U\right)(\chi)+\widetilde{A}(U, \chi)-A(t ; U, \chi)
\end{aligned}
$$

or

$$
\begin{aligned}
& \frac{1}{2} \frac{d}{d t}\|\omega U\|^{2}+\widetilde{A}(\omega U, \omega U) \\
& \leqslant \widetilde{A}(\omega U, \omega U)-\widetilde{A}\left(U, \omega^{2} U\right) \mid+\left\|U_{t}\right\|_{\Omega_{1}}\left\|\omega^{2} U-\chi\right\|_{\Omega_{1}^{\prime}} \\
& +\|U\|_{1, \Omega_{1}^{\prime}}\left\|\widetilde{P}\left(\omega^{2} U\right)-\chi\right\|_{1, \Omega_{1}^{\prime}}+\left(\left\|L_{h} U\right\|_{h,-1, \Omega_{1}}+C\|U\|_{\Omega_{1}}\right)\|\chi\|_{1, \Omega_{1}^{\prime}} .
\end{aligned}
$$

Here we find easily

$$
\widetilde{A}(\omega U, \omega U)-\widetilde{A}\left(U, \omega^{2} U\right)|=| \int_{\Omega_{1}^{\prime}} \sum_{j, k} a_{j k} \frac{\partial \omega}{\partial x_{j}} \frac{\partial \omega}{\partial x_{k}} U^{2} d x \mid \leqslant C\|U\|_{\Omega_{1}}^{2},
$$

and choosing $\chi$ as in Lemma 2.4, we have

$$
\begin{gathered}
\left\|U_{t}\right\|_{\Omega_{1}}\left\|\omega^{2} U-\chi\right\|_{\Omega_{1}^{\prime}}+\|U\|_{1, \Omega_{1}^{\prime}}\left\|\widetilde{P}\left(\omega^{2} U\right)-\chi\right\|_{1, \Omega_{1}^{\prime}} \\
\leqslant C h\|U\|_{\Omega_{1}}\left(\left\|U_{t}\right\|_{\Omega_{1}}+\|U\|_{1, \Omega_{1}^{\prime}}\right) .
\end{gathered}
$$

Further, 


$$
\begin{aligned}
\|\chi\|_{1, \Omega_{1}^{\prime}} & \leqslant\left\|\widetilde{P}\left(\omega^{2} U\right)-\chi\right\|_{1, \Omega_{1}^{\prime}}+\left\|\widetilde{P}\left(\omega^{2} U\right)\right\|_{1, \Omega_{1}^{\prime}} \\
& \leqslant C\left(h\|U\|_{\Omega_{1}}+\left\|\omega^{2} U\right\|_{1, \Omega_{1}^{\prime}}\right) \leqslant C\left(\|U\|_{\Omega_{1}}+\widetilde{A}(\omega U, \omega U)^{1 / 2}\right) .
\end{aligned}
$$

Together, these estimates yield

$$
\begin{aligned}
& \frac{d}{d t}\|\omega U\|^{2}+ 2 \tilde{A}(\omega U, \omega U) \\
& \leqslant C\left\{\|U\|_{\Omega_{1}}^{2}+h\left\|U_{t}\right\|_{\Omega_{1}}\|U\|_{\Omega_{1}}+h\|U\|_{1, \Omega_{1}^{\prime}}\|U\|_{\Omega_{1}}\right. \\
&\left.\quad+\left(\left\|L_{h} U\right\|_{h,-1, \Omega_{1}}+\|U\|_{\Omega_{1}}\right)\left(\tilde{A}(\omega U, \omega U)^{1 / 2}+\|U\|_{\Omega_{1}}\right)\right\} .
\end{aligned}
$$

Using the inverse estimate (a) on the third term on the right, we obtain easily

$$
\frac{d}{d t}\|\omega U\|^{2}+\widetilde{A}(\omega U, \omega U) \leqslant C\left\{\|U\|_{\Omega_{1}}^{2}+h^{2}\left\|U_{t}\right\|_{\Omega_{1}}^{2}+\left\|L_{h} U\right\|_{h,-1, \Omega_{1}}^{2}\right\} .
$$

Integration over $(0, t)$ now completes the proof for $q=2$.

For the purpose of further estimating the term in $U_{t}$ above we shall now prove

LеммA 3.2. Let $\Omega_{2} \subset \subset \Omega_{1} \subset \Omega_{0}$ and let $q$ be an arbitrary number. We then have for $U:[0, T] \rightarrow S_{h}\left(\Omega_{0}\right)$,

$$
\begin{aligned}
& \|U(t)\|_{1, \Omega_{2}}^{2}+\int_{0}^{t}\left\|U_{t}\right\|_{\Omega_{2}}^{2} d \tau \\
& \quad \leqslant C\left\{\|U(0)\|_{1, \Omega_{1}}^{2}+\int_{0}^{t}\left(\|U\|_{1, \Omega_{1}}^{2}+h^{q}\left\|U_{t}\right\|_{\Omega_{1}}^{2}+\left\|L_{h} U\right\|_{h, 0, \Omega_{1}}^{2}\right) d \tau\right\} .
\end{aligned}
$$

Proof. With the notation of the proof of Lemma 3.1, we have now for $\chi \in$ $S_{h}^{0}\left(\Omega_{1}^{\prime}\right)$

$$
\begin{aligned}
& \left(U_{t}, \omega^{2} U_{t}\right)+\widetilde{A}\left(U, \omega^{2} U_{t}\right) \\
& \quad=\left(U_{t}, \omega^{2} U_{t}-\chi\right)+\widetilde{A}\left(U, \widetilde{P}\left(\omega^{2} U_{t}\right)-\chi\right)+\left(L_{h} U\right)(\chi)+(\widetilde{A}(U, \chi)-A(t ; U, \chi)) .
\end{aligned}
$$

Hence

$$
\begin{aligned}
\left\|\omega U_{t}\right\|^{2}+\widetilde{A}\left(\omega U, \omega U_{t}\right) \leqslant & \tilde{A}\left(\omega U, \omega U_{t}\right)-\widetilde{A}\left(U, \omega^{2} U_{t}\right) \mid \\
& +\left\|U_{t}\right\|_{\Omega_{1}}\left\|\omega^{2} U_{t}-\chi\right\|_{\Omega_{1}^{\prime}}+\|U\|_{1, \Omega_{1}}\left\|\widetilde{P}\left(\omega^{2} U_{t}\right)-\chi\right\|_{1, \Omega_{1}^{\prime}} \\
& +\left(\left\|L_{h} U\right\|_{h, 0, \Omega_{1}}+C\|U\|_{1, \Omega_{1}}\right)\|\chi\|_{\Omega_{1}^{\prime}} \cdot
\end{aligned}
$$

We find here

$$
\begin{aligned}
\widetilde{A}(\omega U, & \left.\omega U_{t}\right)-\widetilde{A}\left(U, \omega^{2} U_{t}\right) \mid \\
& =\left|\int_{\Omega_{1}} \sum_{j, k} \omega U_{t}\left[a_{j k} \frac{\partial \omega}{\partial x_{j}} \frac{\partial U}{\partial x_{k}}+\frac{\partial}{\partial x_{j}}\left(a_{j k} \frac{\partial \omega}{\partial x_{k}} U\right)\right] d x\right| \\
& \leqslant C\left\|\omega U_{t}\right\|\|U\|_{1, \Omega_{1}} \leqslant 1 / 4\left\|\omega U_{t}\right\|^{2}+C\|U\|_{1, \Omega_{1}}^{2} .
\end{aligned}
$$


Further, choosing $\chi$ by Lemma 2.4 ,

and

$$
\begin{gathered}
\left\|U_{t}\right\|_{\Omega_{1}}\left\|\omega^{2} U_{t}-\chi\right\|_{\Omega_{1}^{\prime}}+\|U\|_{1, \Omega_{1}}\left\|\widetilde{P}\left(\omega^{2} U_{t}\right)-\chi\right\|_{1, \Omega_{1}^{\prime}} \\
\leqslant C h\left\|U_{t}\right\|_{\Omega_{1}}\left(\left\|U_{t}\right\|_{\Omega_{1}}+\|U\|_{1, \Omega_{1}}\right)
\end{gathered}
$$

$$
\|\chi\|_{\Omega_{1}^{\prime}} \leqslant\left\|\omega^{2} U_{t}-\chi\right\|_{\Omega_{1}^{\prime}}+\left\|\omega^{2} U_{t}\right\| \leqslant C\left(h\left\|U_{t}\right\|_{\Omega_{1}}+\left\|\omega U_{t}\right\|\right) .
$$

Hence, since

$$
\left|\widetilde{A}\left(\omega U, \omega U_{t}\right)-\frac{1}{2} \frac{d}{d t} \widetilde{A}(\omega U, \omega U)\right| \leqslant C\|U\|_{1, \Omega_{1}}^{2},
$$

we obtain

$$
\begin{aligned}
\left\|\omega U_{t}\right\|^{2}+ & \frac{1}{2} \frac{d}{d t} \widetilde{A}(\omega U, \omega U) \\
\leqslant & \frac{1}{4}\left\|\omega U_{t}\right\|^{2}+C\left\{\|U\|_{1, \Omega_{1}}^{2}+h\left\|U_{t}\right\|_{\Omega_{1}}^{2}+h\|U\|_{1, \Omega_{1}}\left\|U_{t}\right\|_{\Omega_{1}}\right. \\
& \left.+\left(\left\|L_{h} U\right\|_{h, 0, \Omega_{1}}+\|U\|_{1, \Omega_{1}}\right)\left(h\left\|U_{t}\right\|_{\Omega_{1}}+\left\|\omega U_{t}\right\|\right)\right\},
\end{aligned}
$$

or by obvious estimates

$$
\left\|\omega U_{t}\right\|^{2}+\frac{d}{d t} \tilde{A}(\omega U, \omega U) \leqslant C\left\{\|U\|_{1, \Omega_{1}}^{2}+h\left\|U_{t}\right\|_{\Omega_{1}}^{2}+\left\|L_{h} U\right\|_{h, 0, \Omega_{1}}^{2}\right\} .
$$

By integration over $(0, t)$ we obtain

$$
\begin{aligned}
& \int_{0}^{t}\left\|U_{t}\right\|_{\Omega_{2}}^{2} d \tau+\|U\|_{1, \Omega_{2}}^{2} \\
& \quad \leqslant C\left\{\|U(0)\|_{1, \Omega_{1}}^{2}+\int_{0}^{t}\left[\|U\|_{1, \Omega_{1}}^{2}+h\left\|U_{t}\right\|_{\Omega_{1}}^{2}+\left\|L_{h} U\right\|_{h, 0, \Omega_{1}}^{2}\right] d \tau\right\},
\end{aligned}
$$

that is, the desired estimate with $q=1$. Repeated application of this estimate, using a sequence of intermediate domains, allows us to increase the power of $h$ to any order, and thus completes the proof of the lemma.

We may now complete the proof of Lemma 3.1. Let $\Omega_{2} \subset \subset \Omega_{1}^{\prime \prime} \subset \subset \Omega_{1}^{\prime} \subset \subset \Omega_{1}$ and note that by the inverse estimate $(a)$,

and

$$
h\|U\|_{1, \Omega_{1}^{\prime}} \leqslant C\|U\|_{\Omega_{1}}
$$

$$
h\left\|L_{h} U\right\|_{h, 0, \Omega_{1}^{\prime}} \leqslant C\left\|L_{h} U\right\|_{h,-1, \Omega_{1}} .
$$

We may, therefore, conclude from Lemma 3.2 that $h^{2} \int_{0}^{t}\left\|U_{t}\right\|_{\Omega_{1}^{\prime \prime}}^{2} d \tau$

$$
\begin{aligned}
& \leqslant C\left\{h^{2}\|U(0)\|_{1, \Omega_{1}^{\prime}}^{2}+\int_{0}^{t}\left[h^{2}\|U\|_{1, \Omega_{1}^{\prime}}^{2}+h^{q}\left\|U_{t}\right\|_{\Omega_{1}}^{2}+h^{2}\left\|L_{h} U\right\|_{h, 0, \Omega_{1}^{\prime}}^{2}\right] d \tau\right\} \\
& \leqslant C\left\{\|U(0)\|_{\Omega_{1}}^{2}+\int_{0}^{t}\left[\|U\|_{\Omega_{1}}^{2}+h^{q}\left\|U_{t}\right\|_{\Omega_{1}}^{2}+\left\|L_{h} U\right\|_{h,-1, \Omega_{1}}^{2}\right] d \tau\right\} .
\end{aligned}
$$


The desired result is now a consequence of the case $q=2$ of Lemma 3.1 which we have already established.

We can now state and prove our main a priori interior estimate for the semidiscrete problem.

Lemma 3.3. Let $\Omega_{2} \subset \subset \Omega_{1} \subset \Omega_{0}$ and let $q$ be arbitrary. Assume that $U:[0, T]$ $\rightarrow S_{h}\left(\Omega_{0}\right)$ satisfies

$$
\left(L_{h} U\right)(\chi) \equiv\left(U_{t}, \chi\right)+A(t ; U, \chi)=(F, \chi) \quad \forall \chi \in S_{h}^{0}\left(\Omega_{0}\right), U(0)=V .
$$

Then for any $m \geqslant 0$,

$$
\begin{aligned}
& \sum_{|\alpha| \leqslant m}\left\|\partial_{h}^{\alpha} U(t)\right\|_{\Omega_{2}}^{2} \\
& \quad \leqslant C\left\{\sum_{|\alpha| \leqslant m}\left\|\partial_{h}^{\alpha} V\right\|_{\Omega_{1}}^{2}+\int_{0}^{t}\left[\|U\|_{\Omega_{1}}^{2}+h^{q}\left\|U_{t}\right\|_{\Omega_{1}}^{2}+\sum_{|\alpha| \leqslant m}\left\|\partial_{h}^{\alpha} F\right\|_{-1, \Omega_{1}}^{2}\right] d \tau\right\}
\end{aligned}
$$

Proof. For $m=0$ this is contained in Lemma 3.1. In order to apply Lemma 3.1 to $\partial_{h}^{\alpha} U$ for $|\alpha|=m>0$, let $\Omega_{2} \subset \subset \Omega_{1}^{\prime} \subset \subset \Omega_{1}$ and notice that for $\chi \in S_{h}^{0}\left(\Omega_{1}^{\prime}\right)$ and small $h$,

$$
\left(L_{h}\left(\partial_{h}^{\alpha} U\right)\right)(\chi)=\left(\partial_{h}^{\alpha} U_{t}, \chi\right)+A\left(t ; \partial_{h}^{\alpha} U, \chi\right)=\left(\partial_{h}^{\alpha} F, \chi\right)+R_{h}^{(\alpha)},
$$

where (with $E_{h}^{\alpha} v=v(\cdot+\alpha h)$ )

$$
\begin{aligned}
R_{h}^{(\alpha)}=-\int_{\Omega_{1}^{\prime}} \sum_{\beta<\alpha}(\alpha-\beta)\{ & \sum_{j, k=1}^{N} \partial_{h}^{\alpha-\beta} a_{j k} E_{h}^{\alpha-\beta} \partial_{h}^{\beta} \frac{\partial U}{\partial x_{j}} \frac{\partial \chi}{\partial x_{k}} \\
& \left.+\sum_{j=1}^{N} \partial_{h}^{\alpha-\beta} a_{j} E_{h}^{\alpha-\beta} \partial_{h}^{\beta} \frac{\partial U}{\partial x_{j}} \chi+\partial_{h}^{\alpha-\beta} a_{0} E_{h}^{\alpha-\beta} \partial_{h}^{\beta} U \chi\right\} d x .
\end{aligned}
$$

We find immediately

$$
\left|R_{h}^{(\alpha)}\right| \leqslant C \sum_{|\beta|<|\alpha|}\left\|\partial_{h}^{\beta} U\right\|_{1, \Omega_{1}}\|\chi\|_{1, \Omega_{1}^{\prime}},
$$

so that

$$
\left\|L_{h}\left(\partial_{h}^{\alpha} U\right)\right\|_{h,-1, \Omega_{1}^{\prime}} \leqslant C\left(\left\|\partial_{h}^{\alpha} F\right\|_{-1, \Omega_{1}}+\sum_{|\beta|<|\alpha|}\left\|\partial_{h}^{\beta} U\right\|_{1, \Omega_{1}}\right) .
$$

Noticing that

and

$$
h^{q+2|\alpha|}\left\|\partial_{h}^{\alpha} U_{t}\right\|_{\Omega_{1}^{\prime}}^{2} \leqslant C h^{q}\left\|U_{t}\right\|_{\Omega_{1}}^{2}
$$

$$
\sum_{|\alpha| \leqslant m}\left\|\partial_{h}^{\alpha} U\right\|_{\Omega_{1}^{\prime}}^{2} \leqslant \sum_{|\alpha| \leqslant m-1}\left\|\partial_{h}^{\alpha} U\right\|_{1, \Omega_{1}}^{2}
$$

we now obtain by application of Lemma 3.1 to $\partial_{h}^{\alpha} U$ for $|\alpha| \leqslant m$, with $q$ replaced by 
$q+2|\alpha|$ and $\Omega_{1}$ by $\Omega_{1}^{\prime}$,

$$
\begin{aligned}
\sum_{|\alpha| \leqslant m}\left[\left\|\partial_{h}^{\alpha} U\right\|_{\Omega_{2}}^{2}\right. & \left.+\int_{0}^{t}\left\|\partial_{h}^{\alpha} U\right\|_{1, \Omega_{2}}^{2} d \tau\right] \\
\leqslant C\left\{\sum_{|\alpha| \leqslant m}\left\|\partial_{h}^{\alpha} V\right\|_{\Omega_{1}}^{2}+\int_{0}^{t}\left[\sum_{|\alpha| \leqslant m-1}\left\|\partial_{h}^{\alpha} U\right\|_{1, \Omega_{1}}^{2}+h^{\alpha}\left\|U_{t}\right\|_{\Omega_{1}}^{2}\right.\right. & \left.\left.+\sum_{|\alpha| \leqslant m}\left\|\partial_{h}^{\alpha} F\right\|_{-1, \Omega_{1}}^{2}\right] d \tau\right\} .
\end{aligned}
$$

By iteration of this inequality we may further reduce the first term of the integrand on the right (using intermediate sets $\Omega_{1}^{\prime}$ with $\Omega_{2} \subset \subset \Omega_{1}^{\prime} \subset \subset \Omega_{1}$ ) to obtain

$$
\begin{aligned}
& \sum_{|\alpha| \leqslant m}\left\|\partial_{h}^{\alpha} U\right\|_{\Omega_{1}}^{2} \\
& \quad \leqslant C\left\{\sum_{|\alpha| \leqslant m}\left\|\partial_{h}^{\alpha} V\right\|_{\Omega_{1}}^{2}+\int_{0}^{t}\left[\|U\|_{1, \Omega_{1}}^{2}+h^{q}\left\|U_{t}\right\|_{\Omega_{1}}^{2}+\sum_{|\alpha| \leqslant m}\left\|\partial_{h} F\right\|_{-1, \Omega_{1}}^{2}\right] d \tau\right\} .
\end{aligned}
$$

The result now follows by a final application of Lemma 3.1 (again using an intermediate set between $\Omega_{2}$ and $\Omega_{1}$ ).

We shall complete this section by deriving a version of the above inequality valid for $t$ bounded away from zero, and with the bound on the right using only function values over a short interval preceding $t$.

LemMA 3.4. Let $\Omega_{2} \subset \subset \Omega_{1} \subset \Omega_{0}$ and let $q$ be arbitrary. Assume that $U$ : $[0, T] \rightarrow S_{h}\left(\Omega_{0}\right)$ satisfies

$$
\left(L_{h} U\right)(\chi)=\left(U_{t}, \chi\right)+A(t ; U, \chi)=(F, \chi) \quad \forall \chi \in S_{h}^{0}\left(\Omega_{0}\right) .
$$

Then for $0<\delta \leqslant t \leqslant T$ and any $m \geqslant 0$,

$$
\sum_{|\alpha| \leqslant m}\left\|\partial_{h}^{\alpha} U(t)\right\|_{\Omega_{2}}^{2} \leqslant C \int_{t-\delta}^{t}\left[\|U\|_{\Omega_{1}}^{2}+h^{q}\left\|U_{t}\right\|_{\Omega_{1}}^{2}+\sum_{|\alpha| \leqslant m}\left\|\partial_{h} F\right\|_{-1, \Omega_{1}}^{2}\right] d \tau .
$$

Proof. Let $\varphi \in C^{1}$ be such that $\varphi(\tau)=1$ for $\tau \geqslant 0, \varphi(\tau)=0$ for $\tau \leqslant-\delta / 2$ and set $\varphi_{0}(\tau)=\varphi(\tau-t)$. We have at time $\tau$,

$$
L_{h}\left(\varphi_{0} U\right)(\chi)=\varphi_{0}\left(L_{h} U\right)(\chi)+\varphi_{0}^{\prime}(U, \chi)=\left(\varphi_{0} F+\varphi_{0}^{\prime} U, \chi\right) \quad \forall \chi \in S_{h}^{0}\left(\Omega_{0}\right) .
$$

Application of Lemma 3.3 hence gives

$$
\begin{aligned}
& \sum_{|\alpha| \leqslant m}\left\|\partial_{h}^{\alpha} U(t)\right\|_{\Omega_{2}}^{2} \\
& \quad \leqslant C \int_{t-\delta / 2}^{t}\left[\sum_{|\alpha| \leqslant \max (m-1,0)}\left\|\partial_{h}^{\alpha} U\right\|_{\Omega_{1}}^{2}+h^{q}\left\|U_{t}\right\|_{\Omega_{1}}^{2}+\sum_{|\alpha| \leqslant m}\left\|\partial_{h}^{\alpha} F\right\|_{-1, \Omega_{1}}^{2}\right] d \tau .
\end{aligned}
$$

The desired result now follows by an easy induction over $m$.

4. The Basic Interior Error Estimates. In this section we shall show the basic results of this paper, the interior maximum-norm estimates for the error in approximating a derivative of the exact solution of the parabolic equation by an appropriate 
finite difference quotient of the semidiscrete solution. The first such estimate below, Theorem 4.1, is interior with respect to the space variables but uniform for $t$ in $[0, T]$ whereas the second result, Theorem 4.2, is interior in both space and time, thus valid for $t$ bounded away from zero. Both error estimates contain one term which is $O\left(h^{r}\right)$ under the appropriate local regularity assumptions and one term containing weak norms of the error in a larger domain. In our applications in Section 5 these latter terms will be majorized by global error bounds. For comparison, we shall finally state in Theorem 4.3 an interior in space estimate derived by a technique used in [2]. The error bound now contains time derivatives of the error.

We shall assume throughout this section without explicit mention that $\left\{S_{h}\right\}$ satisfies the regularity assumptions of Section 2 in the interior domain $\Omega_{0}$. We shall consider the interior equations

$$
L u=u_{t}+A u=f \quad \text { in } \quad \Omega_{0} \times[0, T],
$$

and with $u_{h}:[0, T] \rightarrow S_{h}$,

$$
\left(L_{h} u_{h}\right)(\chi)=\left(u_{h, t}, \chi\right)+A\left(t ; u_{h}, \chi\right)=(f, \chi) \quad \nabla^{\prime} \chi \in S_{h}^{0}\left(\Omega_{0}\right),
$$

respectively.

We shall begin by deriving an interior $L_{2}$-estimate for difference quotients of the error.

Lemma 4.1. Assume that $u$ and $u_{h}$ are solutions of (4.1) and (4.2), respectively, and that $v_{h}=u_{h}(0)$ and $v=u(0)$ satisfy the interior equation

$$
A_{0}\left(v_{h}-v, \chi\right)=0 \quad \forall \chi \in S_{h}^{0}\left(\Omega_{0}\right),
$$

where $A_{0}(\cdot, \cdot)$ is a bilinear form corresponding to a second order elliptic differential operator with smooth coefficients. Let $\Omega_{2} \subset \subset \Omega_{1} \subset \Omega_{0}$ and let $p, q$ be arbitrary and $m$ positive. Then for $e_{h}=u_{h}-u$,

$$
\sum_{|\alpha| \leqslant m}\left\|\partial_{h}^{\alpha} e_{h}(t)\right\|_{\Omega_{2}} \leqslant C\left\{h^{r} B_{r+m, \Omega_{1}}(t ; u)+R_{\Omega_{1}}\left(t ; e_{h}\right)\right\},
$$

where

$$
B_{s, \Omega_{1}}(t ; u)=\sup _{0 \leqslant \tau \leqslant t}\|u(\tau)\|_{s, \Omega_{1}}+\left(\int_{0}^{t}\left\|u_{t}\right\|_{s-1, \Omega_{1}}^{2} d \tau\right)^{1 / 2}
$$

and

$$
R_{\Omega_{1}}\left(t ; e_{h}\right)=\left\|e_{h}(0)\right\|_{-p, \Omega_{1}}+\left(\int_{0}^{t}\left(\left\|e_{h}\right\|_{\Omega_{1}}^{2}+h^{q}\left\|e_{h, t}\right\|_{\Omega_{1}}^{2}\right) d \tau\right)^{1 / 2}
$$

Proof. Let $\widetilde{P}=\widetilde{P}_{\Omega_{1}}(t)$ denote the projection used in Section 2 with respect to $\widetilde{A}(\cdot, \cdot)$, and set $\theta=u_{h}-\widetilde{P} u$ and $\rho=(I-\widetilde{P}) u$. We then have

$$
\left(L_{h} \theta\right)(\chi)=\left(\rho_{t}+\sum_{j} a_{j} \frac{\partial \rho}{\partial x_{j}}+\left(a_{0}-1\right) \rho, \chi\right) \quad \forall \chi \in S_{h}^{0}\left(\Omega_{1}\right),
$$


so that noticing that $\theta \in S_{h}\left(\Omega_{0}\right)$ and letting $\Omega_{2} \subset \subset \Omega_{1}^{\prime} \subset \subset \Omega_{1}$ we may apply Lemma 3.3 to obtain

$$
\begin{aligned}
& \sum_{|\alpha| \leqslant m}\left\|\partial_{h}^{\alpha} \theta\right\|_{\Omega_{2}}^{2} \\
& \leqslant C\left\{\sum_{|\alpha| \leqslant m}\left\|\partial_{h}^{\alpha} \theta(0)\right\|_{\Omega_{1}^{\prime}}^{2}+\int_{0}^{t}\left[\|\theta\|_{\Omega_{1}^{\prime}}^{2}+h^{q}\left\|\theta_{t}\right\|_{\Omega_{1}^{\prime}}^{2}\right.\right. \\
& \left.\left.\quad+\sum_{|\alpha| \leqslant m}\left(\left\|\partial_{h}^{\alpha} \rho\right\|_{\Omega_{1}^{\prime}}^{2}+\left\|\partial_{h}^{\alpha} \rho_{t}\right\|_{-1, \Omega_{1}^{\prime}}^{2}\right)\right] d \tau\right\} .
\end{aligned}
$$

Consider first the term with $\theta(0)=v_{h}-\widetilde{P} v$. We have for $|\alpha| \leqslant m$, applying Lemma 2.2 to $e_{h}(0)$ and Lemma 2.3 to $\rho(0)$,

$$
\begin{aligned}
\left\|\partial_{h}^{\alpha} \theta(0)\right\|_{\Omega_{1}^{\prime}} & \leqslant\left\|\partial_{h} e_{h}(0)\right\|_{\Omega_{1}^{\prime}}+\left\|\partial_{h}^{\alpha} \rho(0)\right\|_{\Omega_{1}^{\prime}} \\
& \leqslant C\left\{h^{r}\|u(0)\|_{r+m, \Omega_{1}}+\left\|e_{h}(0)\right\|_{-p, \Omega_{1}}\right\}
\end{aligned}
$$

which is bounded by the right side of (4.4).

Further, assuming as we may, that $q \geqslant 2$, we have

$$
\begin{aligned}
& \int_{0}^{t}\left[\|\theta\|_{\Omega_{1}^{\prime}}^{2}\right.\left.+h^{q}\left\|\theta_{t}\right\|_{\Omega_{1}^{\prime}}^{2}\right] d \tau \\
& \leqslant C \int_{0}^{t}\left[\left\|e_{h}\right\|_{\Omega_{1}}^{2}+h^{q}\left\|e_{h, t}\right\|_{\Omega_{1}}^{2}+\|\rho\|_{\Omega_{1}}^{2}+h^{2}\left\|\rho_{t}\right\|_{\Omega_{1}}^{2}\right] d \tau .
\end{aligned}
$$

Here, the terms containing $e_{h}$ are bounded by $C \cdot R_{\Omega_{1}}\left(t ; e_{h}\right)^{2}$ and by (2.6) and (2.7) we have

$$
\int_{0}^{t}\left(\|\rho\|_{\Omega_{1}}^{2}+h^{2}\left\|\rho_{t}\right\|_{\Omega_{1}}^{2}\right) d \tau \leqslant C h^{2 r} \int_{0}^{t}\left(\|u\|_{r, \Omega_{1}}^{2}+\left\|u_{t}\right\|_{r-1, \Omega_{1}}^{2}\right) d \tau .
$$

Finally, by Lemma 2.3,

$$
\begin{aligned}
& \int_{0}^{t} \sum_{|\alpha| \leqslant m}\left[\left\|\partial_{h}^{\alpha} \rho\right\|_{\Omega_{1}^{\prime}}^{2}+\left\|\partial_{h}^{\alpha} \rho_{t}\right\|_{-1, \Omega_{1}^{\prime}}^{2}\right] d \tau \\
& \quad \leqslant C h^{2 r} \int_{0}^{t}\left[\|u\|_{r+m, \Omega_{1}}^{2}+\left\|u_{t}\right\|_{r+m-1, \Omega_{1}}^{2}\right] d \tau
\end{aligned}
$$

so that altogether

$$
\sum_{|\alpha| \leqslant m}\left\|\partial_{h}^{\alpha} \theta\right\|_{\Omega_{2}} \leqslant C\left\{h^{r} B_{r+m, \Omega_{1}}(t ; u)+R_{\Omega_{1}}\left(t ; e_{h}\right)\right\} .
$$

Since by Lemma 2.3,

$$
\sum_{|\alpha| \leqslant m}\left\|\partial_{h}^{\alpha} \rho(t)\right\|_{\Omega_{2}} \leqslant C h^{r}\|u(t)\|_{r+m, \Omega_{1}} \leqslant C h^{r} B_{r+m, \Omega_{1}}(t ; u),
$$

and since $e_{h}=\theta-\rho$ the desired result now follows by the triangle inequality.

We are now in a position to establish our first maximum-norm bound for the error between an arbitrary derivative $D^{\alpha} u(|\alpha|=m)$ and a corresponding finite difference 
approximation $Q_{h} u_{h}$. Thus, let $Q_{h}$ be defined, with finitely many constants $q_{\beta \gamma}$, by

$$
Q_{h} w(x)=\sum_{|\beta|=m, \gamma} q_{\beta \gamma} \partial_{h}^{\beta} w(x-\gamma h)
$$

We say that $Q_{h}$ approximates $D^{\alpha}$ with order of accuracy $r$ if

$$
\left|Q_{h} w-D^{\alpha} w\right|_{\Omega_{2}} \leqslant C h^{r}|w|_{r+m, \Omega_{1}} \quad \text { if } \quad \Omega_{2} \subset \subset \Omega_{1} .
$$

We then have

Theorem 4.1. Assume that $u, u_{h}, v=u(0)$ and $v_{h}=u_{h}(0)$ satisfy (4.1), (4.2) and (4.3) and let $Q_{h}$ be a finite difference operator approximating $D^{\alpha}(|\alpha|=m)$ with order of accuracy $r$. Let $\Omega_{2} \subset \subset \Omega_{1} \subset \Omega_{0}$ and let $p, q$ be arbitrary. Then with the notation of Lemma 4.1 we have for $0 \leqslant t \leqslant T\left(N_{0}=[N / 2]+1\right)$,

$$
\left|Q_{h} u_{h}(t)-D^{\alpha} u(t)\right| \leqslant C\left\{h^{r} B_{r+m+N_{0}, \Omega_{1}}(t ; u)+R_{\Omega_{1}}\left(t ; e_{h}\right)\right\} .
$$

Proof. Let $\Omega_{1}^{\prime}$ and $\Omega_{1}^{\prime \prime}$ be such that $\Omega_{2} \subset \subset \Omega_{1}^{\prime \prime} \subset \subset \Omega_{1}^{\prime} \subset \subset \Omega_{1}$. We have by the form of $Q_{h}$ and since $u_{h}-u=\theta-\rho$,

$$
\left|Q_{h} u_{h}(t)-D^{\alpha} u(t)\right|_{\Omega_{2}} \leqslant C \sum_{|\alpha|=m}\left(\left|\partial_{h}^{\alpha} \theta(t)\right|+\left|\partial_{h}^{\alpha} \rho(t)\right|\right)+\left|\left(Q_{h}-D^{\alpha}\right) u(t)\right|_{\Omega_{2}} .
$$

Here by (4.7),

$$
\left|\left(Q_{h}-D^{\alpha}\right) u(t)\right|_{\Omega_{2}} \leqslant C h^{r}|u(t)|_{r+m, \Omega_{1}} \leqslant C h^{r} B_{r+m+N_{0}, \Omega_{1}}(t ; u) .
$$

Using the discrete Sobolev inequality,

$$
|\chi|_{\Omega_{1}^{\prime \prime}} \leqslant C \sum_{|\beta| \leqslant N_{0}}\left\|\partial_{h}^{\beta} \chi\right\|_{\Omega_{1}^{\prime}} \quad \forall \chi \in S_{h}\left(\Omega_{1}\right),
$$

we have by (4.6),

$$
\begin{aligned}
\sum_{|\alpha| \leqslant m}\left|\partial_{h}^{\alpha} \theta(t)\right|_{\Omega_{1}^{\prime \prime}} & \leqslant C \sum_{|\alpha| \leqslant m+N_{0}}\left\|\partial_{h}^{\alpha} \theta(t)\right\|_{\Omega_{1}^{\prime}} \\
& \leqslant C\left\{h^{r} B_{r+m+N_{0}, \Omega_{1}}(t ; u)+R_{\Omega_{1}}\left(t ; e_{h}\right)\right\} .
\end{aligned}
$$

Since finally by Lemma 2.3 ,

$$
\sum_{|\alpha| \leqslant m}\left|\partial_{h}^{\alpha} \rho(t)\right|_{\Omega_{1}^{\prime \prime}} \leqslant C h^{r}\|u(t)\|_{r+m+N_{0}, \Omega_{1}} \leqslant C h^{r} B_{r+m+N_{0}, \Omega_{1}}(t ; u),
$$

the proof is complete.

We shall now deduce an estimate which is interior also with respect to time. The result is now independent of the choice of the initial data and the low order norms of $e_{h}$ in the error bound are taken only over a short interval preceding the time at which the estimate for $Q_{h} u_{h}-D^{\alpha} u$ is given.

THEOREM 4.2. Assume that $u$ and $u_{h}$ satisfy (4.1) and (4.2), respectively, and let $Q_{h}$ be a finite difference operator approximating $D^{\alpha}(|\alpha|=m)$ with order of accuracy $r$. Let $\Omega_{2} \subset \subset \Omega_{1} \subset \Omega_{0}$ and let $p, q$ be arbitrary. Then for $0<\delta \leqslant t \leqslant T$, 
with $e_{h}=u_{h}-u$,

$$
\left|Q_{h} u_{h}(t)-D^{\alpha} u(t)\right|_{\Omega_{2}} \leqslant C\left\{h^{r} B_{r+m+N_{0}, \Omega_{1}}(t-\delta, t ; u)+R_{\Omega_{1}}\left(t-\delta, t ; e_{h}\right)\right\},
$$

where

$$
B_{s}(t-\delta, t ; u)=\sup _{t-\delta \leqslant \tau \leqslant t}\|u(\tau)\|_{s, \Omega_{1}}+\left(\int_{t-\delta}^{t}\left\|u_{t}\right\|_{s-1, \Omega_{1}}^{2} d \tau\right)^{1 / 2}
$$

and

$$
R_{\Omega_{1}}\left(t-\delta, t ; e_{h}\right)=\left(\int_{t-\delta}^{t}\left(\left\|e_{h}\right\|_{\Omega_{1}}^{2}+h^{q}\left\|e_{h, t}\right\|_{\Omega_{1}}^{2}\right) d \tau\right)^{1 / 2}
$$

Proof. First, we prove in exactly the same way as in Lemma 4.1, using only Lemma 3.4 instead of Lemma 3.3, that for $m>0$,

$$
\sum_{|\alpha| \leqslant m}\left\|\partial_{h}^{\alpha} e_{h}(t)\right\|_{\Omega_{2}} \leqslant C\left\{h^{r} B_{r+m, \Omega_{1}}(t-\delta, t ; u)+R_{\Omega_{1}}\left(t-\delta, t ; e_{h}\right)\right\}
$$

The proof is then completed as in Theorem 4.1.

We shall now state, for comparison, the result obtained by the technique used for the homogeneous equation in [2], consisting essentially in considering the time derivative in the parabolic equation as a forcing term in an elliptic equation. The result thus derived is weaker than the ones just proved in that the error bound now contains derivatives of $e_{h}$ with respect to time. In our applications in Section 5 this will require stronger regularity properties of $u$ than our previous results. In order to make the reference to the proof in [2] simple we shall therefore content ourselves with formulating the result in the generality concerning the operator $A$ employed in [2], and in Section 5 below.

Theorem 4.3. Assume that $A$ is time-independent, selfadjoint and nonnegative $\left(a_{0} \geqslant 0\right)$ and let $u$ and $u_{h}$ be solutions of (4.1) and (4.2), respectively. Let $Q_{h}$ be a finite difference operator approximating $D^{\alpha}(|\alpha|=m)$ with order of accuracy $r$. Then if $\Omega_{2} \subset \subset \Omega_{1} \subset \Omega_{0}$ we have for $0 \leqslant t \leqslant T$, with $e_{h}=u_{h}-u$,

$$
\begin{aligned}
& \left|Q_{h} u_{h}(t)-D^{\alpha} u(t)\right|_{\Omega_{2}} \\
& \quad \leqslant C\left\{h^{r} \sum_{2 l \leqslant m+N_{0}}\left\|D_{t}^{l} u(t)\right\|_{r+m+N_{0}-2 l, \Omega_{1}}+\sum_{2 l \leqslant m+N_{0}+2}\left\|D_{t}^{l} e_{h}(t)\right\|_{\Omega_{1}}\right\} .
\end{aligned}
$$

Proof. This is proved step by step as [2, Theorem 6.1] with the obvious modifications due to the fact that the equation may now be nonhomogeneous.

5. Some Global Error Estimates for a Class of Time-Independent Problems. In this section we shall supply the global error estimates needed for bounding the terms in $\ddot{e}_{h}$ in Theorems 4.1 through 4.3 in the special case of the generalization to nonhomogeneous equations of the theory developed in [2] for homogeneous parabolic equations. These estimates will all be of order $O\left(h^{r}\right)$ for sufficiently smooth data, and 
application of the results of Section 4 will thus show this order of interior convergence of difference quotients of the approximate solution to derivatives of the exact solution. We shall leave to the reader to combine the regularity requirements of the global estimates below with those of our previous interior results.

Consider thus the initial-boundary value problem

$$
\begin{aligned}
& u_{t}+A u=f \text { in } \Omega \times[0, T] \\
& u=0 \text { on } \partial \Omega \times[0, T], \\
& u(0)=v,
\end{aligned}
$$

where $A$ is now a selfadjoint, nonnegative second order uniformly elliptic differential operator with smooth coefficients, independent of time. Let $\left\{S_{h}\right\}$ be a family of finite dimensional subspaces of $H^{1}(\Omega)$ and assume that corresponding to each $S_{h}$ we are given an approximate solution operator $T_{h}: L_{2}(\Omega) \rightarrow S_{h}$ of the corresponding elliptic problem

$$
A u=f \quad \text { in } \Omega, \quad u=0 \text { on } \partial \Omega,
$$

such that

(i) $T_{h}$ is selfadjoint, positive semidefinite on $L_{2}(\Omega)$ and positive definite on $S_{h}$; and with $T=A^{-1}$ and some $r \geqslant 2$,

(ii) There is a constant $C$ such that

$$
\left\|\left(T_{h}-T\right) f\right\| \leqslant C h^{s+2}\|f\|_{s}, \quad f \in H^{s}(\Omega), 0 \leqslant s \leqslant r-2 .
$$

Consider now the approximate semidiscrete problem of finding $u_{h}:[0, T] \rightarrow$ $S_{h}$ such that

$$
\begin{aligned}
T_{h} u_{h, t}+u_{h} & =T_{h} f \quad \text { for } \quad t \in[0, T] \\
u_{h}(0) & =v_{h} \in S_{h},
\end{aligned}
$$

where $v_{h}$ is some approximation of $v$. Clearly, since $T_{h}$ is positive definite on $S_{h}, u_{h}$ is well defined by (5.2) for $t>0$.

If $\left\{S_{h}\right\}$ satisfies certain standard approximation properties and if the elements of $S_{h}$ vanish on $\partial \Omega$, operators satisfying conditions of types (i) and (ii) may be defined by the ordinary Galerkin equation

$$
A\left(T_{h} f, \chi\right)=(f, \chi) \quad \forall \chi \in S_{h} ;
$$

the semidiscrete equation may then be written

$$
\left(u_{h, t}, \chi\right)+A\left(u_{h}, \chi\right)=(f, \chi) \quad \forall \chi \in S_{h} .
$$

An important feature of the present formulation is that it encompasses also several different other procedures for dealing with the homogeneous boundary conditions (cf. [2]).

If in addition to (i) and (ii) we demand that the family $\left\{S_{h}\right\}$ satisfies the regularity assumptions of Section 2 on the interior subdomain $\Omega_{0}$ of $\Omega$, and that the 
operator $T_{h}$ is such that

$$
A\left(T_{h} f, \chi\right)=(f, \chi) \quad \forall \chi \in S_{h}^{0}\left(\Omega_{0}\right),
$$

so that the semidiscrete interior equation

$$
\left(u_{h, t}, \chi\right)+A\left(u_{h}, \chi\right)=(f, \chi) \quad \forall \chi \in S_{h}^{0}\left(\Omega_{0}\right)
$$

holds for the solution of (5.2), then the results of Section 4 all apply in the present context. The property (iii) is shared by several of the methods referred to above satisfying (i) and (ii).

In the first part of this section we shall assume that $v \in \stackrel{\circ}{H}^{1}(\Omega) \cap H^{2}(\Omega)$ and use for the initial-values $v_{h}$ of $u_{h}$ the elliptic projection of $v$ defined by

$$
v_{h}=P_{1} v=T_{h} A v \text {. }
$$

Recall that for $0 \leqslant s \leqslant r-2$,

$$
\left\|\left(P_{1}-I\right) v\right\|_{-s}=\left\|\left(T_{h}-T\right) A v\right\|_{-s} \leqslant C h^{r}\|v\|_{r-s} .
$$

In fact, we have by (ii),

$$
\left\|\left(T_{h}-T\right) f\right\|_{0} \leqslant C h^{r}\|f\|_{r-2},
$$

and hence, since $T_{h}-T$ is selfadjoint,

$$
\left\|\left(T_{h}-T\right) f\right\|_{-(r-2)}=\sup _{\varphi \in C_{0}^{\infty}(\Omega)} \frac{\left|\left(\left(T_{h}-T\right) f, \varphi\right)\right|}{\|\varphi\|_{r-2}} \leqslant C h^{r}\|f\|_{0} .
$$

We conclude by interpolation for $0 \leqslant s \leqslant r-2$,

$$
\left\|\left(T_{h}-T\right) f\right\|_{-s} \leqslant C h^{r}\|f\|_{r-2-s}
$$

from which (5.3) follows for $f=A v$.

We shall now derive the following global error estimate for the nonhomogeneous equation.

LEMMA 5.1. Under the above assumptions, we have for $e_{h}=u_{h}-u$,

$$
\left(\int_{0}^{t}\left\|e_{h}\right\|^{2} d \tau\right)^{1 / 2} \leqslant C h^{r}\left\{\|v\|_{\max (r-1,2)}+\left(\int_{0}^{t}\|u\|_{r}^{2} d \tau\right)^{1 / 2}\right\} .
$$

Proof. We notice (cf. [2]) that the error satisfies the equation

$$
T_{h} e_{h, t}+e_{h}=\rho \equiv\left(T-T_{h}\right) A u=\left(I-P_{1}\right) u \text { for } t \geqslant 0 .
$$

This implies immediately

$$
\frac{1}{2} \frac{d}{d t}\left(T_{h} e_{h}, e_{h}\right)+\left\|e_{h}\right\|^{2}=\left(\rho, e_{h}\right) \leqslant \frac{1}{2}\|\rho\|^{2}+\frac{1}{2}\left\|e_{h}\right\|^{2},
$$

and hence by integration

$$
\int_{0}^{t}\left\|e_{h}\right\|^{2} d \tau \leqslant\left(T_{h} e_{h}(0), e_{h}(0)\right)+\int_{0}^{t}\|\rho\|^{2} d \tau .
$$


Here, using the boundedness of $T: H^{-1}(\Omega) \rightarrow \stackrel{\circ}{H}^{1}(\Omega)$, and the property (ii) we have

$$
\left(T_{h} w, w\right)=(T w, w)+\left(\left(T_{h}-T\right) w, w\right) \leqslant C\|w\|_{-1}^{2}+C h^{2}\|w\|^{2},
$$

and hence in view of (5.3),

$$
\left(T_{h} e_{h}(0), e_{h}(0)\right) \leqslant C h^{2 r}\|v\|_{\max (r-1,2)}^{2}
$$

Further, by (ii),

$$
\int_{0}^{t}\|\rho\|^{2} d \tau=\int_{0}^{t}\left\|\left(T-T_{h}\right) A u\right\|^{2} d \tau \leqslant C h^{2 r} \int_{0}^{t}\|u\|_{r}^{2} d \tau .
$$

Together these estimates prove the lemma.

We shall now consider the boundedness of $e_{h, t}$.

LEMMA 5.2. Under the above assumptions, we have for $e_{h}=u_{h}-u$,

$$
\left(\int_{0}^{t}\left\|e_{h, t}\right\|^{2} d \tau\right)^{1 / 2} \leqslant C\left\{\|v\|_{2}+\left(\int_{0}^{t}\|f\|^{2} d \tau\right)^{1 / 2}\right\} .
$$

Proof: We have for the exact solution

$$
\left\|u_{t}\right\|^{2}+\frac{1}{2} \frac{d}{d t} A(u, u)=\left(f, u_{t}\right) \leqslant \frac{1}{2}\|f\|^{2}+\frac{1}{2}\left\|u_{t}\right\|^{2}
$$

and hence

$$
\int_{0}^{t}\left\|u_{t}\right\|^{2} d \tau \leqslant A(v, v)+\int_{0}^{t}\|f\|^{2} d \tau \leqslant C\left\{\|v\|_{1}^{2}+\int_{0}^{t}\|f\|^{2} d \tau\right\} .
$$

Similarly, with $A_{h}=T_{h}^{-1}$ on $S_{h}$,

$$
\left\|u_{h, t}\right\|^{2}+\frac{1}{2} \frac{d}{d t}\left(A_{h} u_{h}, u_{h}\right)=\left(f, u_{h, t}\right) \leqslant \frac{1}{2}\|f\|^{2}+\frac{1}{2}\left\|u_{h, t}\right\|^{2},
$$

so that

$$
\int_{0}^{t}\left\|u_{h, t}\right\|^{2} d \tau \leqslant\left(A_{h} v_{h}, v_{h}\right)+\int_{0}^{t}\|f\|^{2} d \tau .
$$

Here $A_{h} v_{h}=A_{h} T_{h} A v=P_{0} A v$ so that by the boundedness of $T_{h}$,

$$
\left(A_{h} v_{h}, v_{h}\right)=\left(A v, T_{h} A v\right) \leqslant C\|A v\|^{2} \leqslant C\|v\|_{2}^{2} .
$$

The result, therefore, follows by (5.5), (5.6), (5.7) and the triangle inequality.

Combination of these results now implies the following estimate suitable for combination with Theorem 4.1.

THEOREM 5.1. Assume that (i) and (ii) hold and let $u$ and $u_{h}$ be the solutions of (5.1) and (5.2), respectively, with $v_{h}=P_{1} v$. Then, with $p=r-2, q=2 r$ in (4.5), we have for $e_{h}=u_{h}-u$,

$$
\begin{aligned}
R_{\Omega_{1}}\left(t ; e_{h}\right) & \leqslant\left\|e_{h}(0)\right\|_{-(r-2)}+\left(\int_{0}^{t}\left(\left\|e_{h}\right\|^{2}+h^{2 r}\left\|e_{h, t}\right\|^{2}\right) d \tau\right)^{1 / 2} \\
& \leqslant C h^{r}\left\{\|v\|_{\max (r-1,2)}+\left(\int_{0}^{t}\left(\|u\|_{r}^{2}+\|f\|^{2}\right) d \tau\right)^{1 / 2}\right\}
\end{aligned}
$$


Proof. We have by (5.3),

$$
\left\|e_{h}(0)\right\|_{-(r-2)}=\left\|\left(P_{1}-I\right) v\right\|_{-(r-2)} \leqslant C h^{r}\|v\|_{2},
$$

and by Lemmas 5.1 and 5.2 ,

$$
\begin{aligned}
\int_{0}^{t}\left(\left\|e_{h}\right\|^{2}\right. & \left.+h^{2 r}\left\|e_{h, t}\right\|^{2}\right) d \tau \\
& \leqslant C h^{2 r}\left\{\|v\|_{\max (r-1,2)}^{2}+\int_{0}^{t}\left(\|u\|_{r}^{2}+\|f\|^{2}\right) d \tau\right\} .
\end{aligned}
$$

Together, these estimates prove the theorem.

Combined with Theorem 4.1 we may conclude, under the appropriate assumptions, that for $\Omega_{2} \subset \subset \Omega_{1} \subset \Omega_{0}$,

$$
\begin{aligned}
& \left|Q_{h} u_{h}(t)-D^{\alpha} u(t)\right|_{\Omega_{2}} \\
& \quad \leqslant C h^{r}\left\{\|v\|_{\max (r-1,2)}+\left(\int_{0}^{t}\left(\|u\|_{r}^{2}+\|f\|^{2}\right) d \tau\right)^{1 / 2}+B_{r+m+N_{0}, \Omega_{1}}(t ; u)\right\} .
\end{aligned}
$$

The regularity demands on $u$ in order that the right-hand side of this inequality be finite can also be expressed exclusively in terms of the data $f$ and $v$ of the problem (cf. e.g. [8]). In addition to regularity of these functions one then also has to impose compatibility conditions between them and the differential operator at $\partial \Omega$ for $t=0$.

We shall now show, using the results of [2] that if we are content with error estimates for time bounded away from zero, then the regularity demands for a $O\left(h^{r}\right)$ result reduce considerably. In such a case, in order to derive the estimate for $Q_{h} u_{h}$ - $D^{\alpha} u$ at time $t$ we shall only have to require strong regularity for a short time preceding $t$ and the compatibility requirements at $\partial \Omega \times\{t=0\}$ disappear. We shall also have more freedom in the choice of approximate initial-values $v_{h}$; we need to require only that $v-v_{h}=O\left(h^{r}\right)$ in some negative norm and that $v_{h}$ is bounded in $L_{2}$. As examples, we notice

$$
\left\|v-P_{0} v\right\|_{-r}+h^{r}\left\|P_{0} v\right\| \leqslant C h^{r}\|v\|
$$

and (cf. [2]),

$$
\left\|v-P_{1} v\right\|_{-(r-2)}+h^{r}\left\|P_{1} v\right\| \leqslant C h^{r}\|v\|_{2} .
$$

We begin by recalling the relevant result from [2], the following global error estimate for the homgeneous equation [2, Theorem 3.3].

LEMmA 5.3. Assume that (i) and (ii) hold and that $u$ and $u_{h}$ are the solutions of (5.1) and (5.2) with $f=0$. Then for $l, p$ nonnegative and arbitrary we have for $e_{h}=u_{h}-u\left(\right.$ with $\left.D_{t}=\partial / \partial t\right)$,

$$
\left\|D_{t}^{l} e_{h}(t)\right\| \leqslant C\left(h^{r} t^{-r / 2-l}\left\|v_{h}\right\|+t^{-p / 2-l}\left\|v_{h}-v\right\|_{-p}\right) .
$$

We are now in a position to show the following global in space, interior in time error estimate, which is what is needed for application of Theorem 4.2. 
THEOREM 5.2. Assume that (i) and (ii) hold and let $u$ and $u_{h}$ be the solutions of (5.1) and (5.2), respectively, with $v_{h}$ arbitrary. Then with $q=2 r$ in (4.8), we have for arbitrary $p, 0<\delta \leqslant t \leqslant T$, and $e_{h}=u_{h}-u$,

$$
\begin{aligned}
& R_{\Omega_{1}}\left(t-\delta / 2, t ; e_{h}\right) \leqslant\left(\int_{t-\delta / 2}^{t}\left(\left\|e_{h}\right\|^{2}+h^{2 r}\left\|e_{h, t}\right\|^{2}\right) d \tau\right)^{1 / 2} \\
& \leqslant C h^{r}\left\{\left\|v_{h}\right\|+\|v\|+h^{-r}\left\|v-v_{h}\right\|_{-p}\right. \\
&\left.+\left(\int_{t-\delta}^{t}\left(\|u\|_{r}^{2}+\|f\|^{2}\right) d \tau\right)^{1 / 2}+\int_{0}^{t}\|f\| d \tau\right\} .
\end{aligned}
$$

Proof. We shall consider a fixed $t=t_{1} \geqslant \delta$. Let $\varphi \in C^{\infty}$ be such that $\varphi(t)$ $=1$ for $t \geqslant-3 \delta / 4, \varphi(t)=0$ for $t \leqslant-\delta$. Set $\varphi_{1}(t)=\varphi\left(t-t_{1}\right)$. We now write $u=u_{1}+u_{2}+u_{3}$ where $u_{1}=u \varphi_{1}$ and $u_{2}$ is the solution of the homogeneous equation,

$$
L u_{2}=0 \text { for } t \geqslant 0, u_{2}(0)=v \text {. }
$$

Since

$$
L u_{1}=f_{1} \equiv f \varphi_{1}+u \varphi_{1}^{\prime} \text { for } t \geqslant 0, u_{1}(0)=0
$$

it follows that $u_{3}$ satisfies

$$
L u_{3}=f_{3} \equiv f\left(1-\varphi_{1}\right)-u \varphi_{1}^{\prime} \quad \text { for } \quad t \geqslant 0, u_{3}(0)=0 .
$$

We notice that $f_{1}$ and $f_{3}$ vanish for $t \leqslant t_{1}-\delta$ and $t \geqslant t_{1}-3 \delta / 4$, respectively.

Let $u_{j, h}, j=1,2,3$, be the semidiscrete approximations of problems (5.11), (5.10), (5.12) with $u_{1, h}(0)=u_{3, h}(0)=0, u_{2, h}(0)=v_{h}$ and set $e_{j, h}=u_{j, h}-u_{j}$. Since by linearity $e_{h}=u_{h}-u=\Sigma_{j} e_{j, h}$ it hence suffices to estimate $R_{\Omega_{1}}\left(t_{1}-\delta / 2, t_{1} ; e_{j, h}\right), j=1,2,3$, by the right-hand side of (5.9) (with $t$ replaced by $t_{1}$ ).

Consider first $u_{1}$. We have by Theorem 5.1, using the definitions of $u_{1}$ and $f_{1}$,

$$
\begin{aligned}
R_{\Omega_{1}}\left(t_{1}-\delta / 2, t_{1} ; e_{1, h}\right)^{2} & \leqslant R_{\Omega_{1}}\left(t_{1} ; e_{1, h}\right)^{2} \leqslant C \int_{0}^{t_{1}}\left(\left\|u_{1}\right\|_{r}^{2}+\left\|f_{1}\right\|^{2}\right) d \tau \\
& \leqslant C \int_{t_{1}-\delta}^{t_{1}}\left(\|u\|_{r}^{2}+\|f\|^{2}\right) d \tau .
\end{aligned}
$$

For $u_{2}$, the solution of the homogeneous equation, we have by Lemma 5.3,

$$
\left\|D_{t}^{l} e_{2, h}\left(t_{1}\right)\right\| \leqslant C\left\{h^{r}\left\|v_{h}\right\|+\left\|v-v_{h}\right\|_{-p}\right\} \text { for } l \geqslant 0
$$

so that in particular

$$
R_{\Omega_{1}}\left(t_{1}-\delta / 2, t_{1} ; e_{2, h}\right) \leqslant C\left\{h^{r}\left\|v_{h}\right\|+\left\|v-v_{h}\right\|_{-p}\right\} .
$$

For the purpose of dealing with $u_{3}$, finally, we introduce the solution operators $E(t)$ and $E_{h}(t)$ of the initial-value problems for the homogeneous exact and semidis- 
crete equations. Setting $F_{h}(t)=E_{h}(t) P_{0}-E(t)$, we notice that Lemma 5.3 implies, by choosing $v_{h}=P_{0} v$ (cf. (5.8)),

$$
\left\|D_{t}^{l} F_{h}(t) v\right\| \leqslant C h^{r}\|v\| \text { for } t \geqslant \delta / 4
$$

We observe now that by superposition we may write, for $t \geqslant t_{1}-\delta / 2$,

$$
e_{3, h}(t)=\int_{0}^{t} F_{h}(t-\tau) f_{3}(\tau) d \tau=\int_{0}^{t_{1}-3 \delta / 4} F_{h}(t-\tau) f_{3}(\tau) d \tau
$$

and also

$$
D_{t}^{l} e_{3, h}(t)=\int_{0}^{t_{1}-3 \delta / 4} D_{t}^{l} F_{h}(t-\tau) f_{3}(\tau) d \tau \text { for } l \geqslant 0
$$

so that for $t \geqslant t_{1}-\delta / 2$,

$$
\left\|D_{t}^{l} e_{3, h}(t)\right\| \leqslant C h^{r} \int_{0}^{t_{1}-3 \delta / 4}\left\|f_{3}\right\| d \tau
$$

$$
\leqslant C h^{r} \int_{0}^{t_{1}}(\|f\|+\|u\|) d \tau \leqslant C h^{r}\left(\|v\|+\int_{0}^{t_{1}}\|f\| d \tau\right)
$$

Here the last step follows by the fact that

$$
\frac{1}{2} \frac{d}{d t}\|u\|^{2}+A(u, u)=(f, u) \leqslant\|f\|\|u\|
$$

implies

$$
\frac{d}{d t}\|u\| \leqslant\|f\|
$$

and hence for $t \geqslant 0$,

$$
\|u(t)\| \leqslant\|v\|+\int_{0}^{t}\|f\| d \tau .
$$

It follows in particular from (5.14) that

$$
R_{\Omega_{1}}\left(t_{1}-\delta / 2, t_{1} ; e_{3, h}\right) \leqslant C h^{r}\left\{\|v\|+\int_{0}^{t_{1}}\|f\| d \tau\right\}
$$

which completes the proof of the theorem.

For the purpose of deriving finally the error estimates for time derivatives needed for application of Theorem 4.3 we shall first show the following error bound for the nonhomogeneous equation.

LEMMA 5.5. Assume that (i) and (ii) hold and that $u$ and $u_{h}$ are the solutions of the problems (5.1) and (5.2). Let $v_{h}=P_{1} v=T_{h} A v$. Then for $e_{h}=u_{h}-u$,

$$
\left\|e_{h}(t)\right\| \leqslant C h^{r}\left\{\|u(t)\|+\left(\int_{0}^{t}\left\|u_{t}\right\|_{\max (r-1,2)}^{2} d \tau\right)^{1 / 2}\right\} .
$$

Proof. Setting $\theta=e_{h}-\rho$, we have by the error equation (cf. Lemma 5.1),

$$
T_{h} \theta_{t}+\theta=-T_{h} \rho_{t}
$$


After multiplication by $\theta_{t}$ we obtain by Schwarz's inequality

$$
\left(T_{h} \theta_{t}, \theta_{t}\right)+\frac{1}{2} \frac{d}{d t}\|\theta\|^{2}=-\left(T_{h} \rho_{t}, \theta_{t}\right) \leqslant \frac{1}{2}\left(T_{h} \rho_{t}, \rho_{t}\right)+\frac{1}{2}\left(T_{h} \theta_{t}, \theta_{t}\right),
$$

so that after integration, since $\theta(0)=0$,

$$
\|\theta(t)\|^{2} \leqslant \int_{0}^{t}\left(T_{h} \rho_{t}, \rho_{t}\right) d \tau .
$$

As in (5.4), (5.3) we obtain, noticing that the elliptic projection commutes with timedifferentiation,

$$
\left(T_{h} \rho_{t}, \rho_{t}\right) \leqslant C\left\{\left\|\rho_{t}\right\|_{-1}^{2}+h^{2}\left\|\rho_{t}\right\|^{2}\right\} \leqslant C h^{2 r}\left\|u_{t}\right\|_{\max (r-1,2)}^{2},
$$

implying

$$
\|\theta(t)\|^{2} \leqslant C h^{2 r} \int_{0}^{t}\left\|u_{t}\right\|_{\max (r-1,2)}^{2} d \tau .
$$

This proves the lemma since

$$
\|\rho(t)\| \leqslant C h^{r}\|u(t)\|_{r}
$$

As a result, we may now establish the following global in space, interior in time error estimates for time derivatives.

THEOREM 5.3. Assume that (i) and (ii) hold and let $u$ and $u_{h}$ be the solutions of (5.1) and (5.2), respectively, with $v_{h}$ arbitrary. Then we have for arbitrary nonnegative $l, p, 0<\delta \leqslant t \leqslant T$, and $e_{h}=u_{h}-u$,

$$
\begin{aligned}
\left\|D_{t}^{l} e_{h}(t)\right\| \leqslant C h^{r}\left\{\left\|v_{h}\right\|\right. & +\|v\|+h^{-r}\left\|v-v_{h}\right\|_{-p}+\left\|D_{t}^{l} u(t)\right\| \\
& \left.+\left(\int_{t-\delta}^{t} \sum_{j=0}^{l+1}\left\|D_{t}^{j} u\right\|_{\max (r-1,2)}^{2} d \tau\right)^{1 / 2}+\int_{0}^{t}\|f\| d \tau\right\} .
\end{aligned}
$$

Proof. Consider again a fixed $t=t_{1} \geqslant \delta$ and decompose the problem into problems (5.11), (5.10) and (5.12) as in the proof of Theorem 5.2. The latter two problems are treated exactly as before and we obtain by (5.13) and (5.14) for $t_{1} \geqslant$ $\delta>0, l \geqslant 0$,

$$
\left\|D_{t}^{l} e_{2, h}\left(t_{1}\right)\right\| \leqslant C\left\{h^{r}\left\|v_{h}\right\|+\left\|v-v_{h}\right\|_{-p}\right\}
$$

and

$$
\left\|D_{t}^{l} e_{3, h}\left(t_{1}\right)\right\| \leqslant C h^{r}\left(\|v\|+\int_{0}^{t_{1}}\|f\| d \tau\right) .
$$

It remains to consider $u_{1}=u \varphi_{1}$. We notice then that for any $l, D_{t}^{l} u_{1}$ and $D_{t}^{l} u_{1, h}$ satisfy equations of the form (5.1) and (5.2), respectively, with $D_{t}^{l} u_{1, h}(0)=P_{1} D_{t}^{l} u_{1}(0)$ $=0$. We may, therefore, apply Lemma 5.4 to obtain

$$
\begin{aligned}
\left\|D_{t}^{l} e_{1, h}\left(t_{1}\right)\right\| & \leqslant C^{r}\left\{\left\|D_{t}^{l} u_{1}\left(t_{1}\right)\right\|+\left(\int_{0}^{t_{1}}\left\|D_{t}^{l+1} u_{1}\right\|_{\max (r-1,2)}^{2} d \tau\right)^{1 / 2}\right\} \\
& \leqslant C h^{r}\left\{\left\|D_{t}^{l} u\left(t_{1}\right)\right\|+\left(\int_{t_{1}-\delta}^{t_{1}} \sum_{j=0}^{l+1}\left\|D_{t}^{j} u\right\|_{\max (r-1,2)}^{2} d \tau\right)^{1 / 2}\right\} .
\end{aligned}
$$

Together these estimates prove the theorem. 
Clearly, the estimate obtained by combination of Theorems 4.3 and 5.3 consumes more global regularity in space than the estimate derived by Theorems 4.2 and 5.2.

Department of Mathematics

Chalmers University of Technology and the University of Göteborg

Fack, S-402 20 Göteborg 5, Sweden

1. J. H. BRAMBLE, J. A. NITSCHE \& A. H. SCHATZ, "Maximum norm interior estimates for Ritz-Galerkin methods," Math. Comp., v. 29, 1975, pp. 677-688.

2. J. H. BRAMBLE, A. H. SCHATZ, V. THOMÉE \& L. B. WAHLBIN, "Some convergence estimates for Galerkin type approximations for parabolic equations," SIAM J. Numer. Anal., v. 14, 1977, pp. 218-241.

3. J. DOUGLAS, JR. \& T. DUPONT, "Galerkin methods for parabolic equations," SIAM J. Numer. Anal., v. 7, 1970, pp. 575-626.

4. J. DOUGLAS, JR. \& T. DUPONT, "Galerkin methods for parabolic equations with nonlinear boundary conditions," Numer. Math., v. 20, 1973, pp. 213-237.

5. T. DUPONT, "Some $L^{2}$ error estimates for parabolic Galerkin methods," The Mathematical Foundations of the Finite Element Method with Applications to Partial Differential Equations (A. K. Aziz, Editor), Academic Press, New York, 1972, pp. 491-501.

6. G. FIX \& N. NASSIF, "On finite element approximations to time dependent problems," Numer. Math., v. 19, 1972, pp. 127-135.

7. H.-P. HELFRICH, Lokale Konvergenz des Galerkinverfahrens bei Gleichungen vom parabolischen Typ in Hilberträumen, Habilitationsschrift, Freiburg, 1975.

8. J. L. LIONS, \& E. MAGENES, Non-Homogeneous Boundary Value Problems and Applications, vol. 1, Springer-Verlag, Berlin and New York, 1972.

9. J. A. NITSCHE \& A. H. SCHATZ, "Interior estimates for Ritz-Galerkin methods," Math. Comp., v. 28, 1974, pp. 937-958.

10. H. S. PRICE \& R. S. VARGA, "Error bounds for semi-discrete Galerkin approximations of parabolic problems with application to petroleum reservoir mechanics," Numerical Solution of Field Problems in Continuum Physics, SIAM-AMS Proc., vol. 11, Amer. Math. Soc., Providence, R. I., 1970, pp. 74-94.

11. M. F. WHEELER, "A priori $L_{2}$ error estimates for Galerkin approximations to parabolic partial differential equations," SIAM J. Numer. Anal., v. 10, 1973, pp. 723-759. 